\title{
PROBLEMS IN HARMONIC ANALYSIS RELATED TO CURVATURE
}

\author{
BY ELIAS M. STEIN AND STEPHEN WAINGER ${ }^{1}$
}

\section{PART I-SURVEY}

1. Preface. Our point of departure for this paper is the standard real variable theorem of Lebesgue on the differentiation of integrals of functions of several variables. Lebesgue's theorem asserts that for any locally integrable function $f$ defined on $R^{n}$

$$
\lim _{\varepsilon \rightarrow 0} \frac{1}{|B(\varepsilon, x)|} \int_{B(\varepsilon, x)} f(y) d y=f(x) \text { a.e., }
$$

for certain types of $n$-dimensional sets $B(\varepsilon, x)$ shrinking to $x$ as $\varepsilon \rightarrow 0$. $(|B(\varepsilon, x)|$ is of course the Lebesgue measure of $B(\varepsilon, x)$.) Some standard examples of sets $B(\varepsilon, x)$ are balls with center $x$ and radius $\varepsilon$, and cubes with center $x$ and diameter $\varepsilon$.

The problem of considering other sets besides balls and cubes for $B(\varepsilon, x)$ received much attention in the 1930's. (See for example Buseman and Feller [1934].) This subject again seems to be attracting interest.

One of the two main goals of this paper is an exposition of recent developments in which $B(\varepsilon, x)$ is replaced by a lower dimensional set. ${ }^{2}$ Specifically we shall examine two cases:

(i) $B(\varepsilon, x)$ is replaced by a sphere with center at $x$ and radius $\varepsilon$,

(ii) $B(\varepsilon, x)$ is replaced by a piece of a curve emanating from $x$.

Thus we ask, does

$$
\lim _{\varepsilon \rightarrow 0} \int_{|y|=1} f(x-\varepsilon y) d \sigma(y)=f(x) \text { a.e.? }
$$

Here $d \sigma$ is the rotationally invariant measure on the sphere $|y|=1$ having total mass 1 . For continuous $f$ the answer is clearly yes. For discontinuous $f$ it is not even clear that $\int f(x-\varepsilon y) d \sigma(y)$ is well defined for all small $\varepsilon$ for almost every $x$. In one dimension the answer to 1 is clearly no, even if $f$ is restricted to lie in the class of bounded functions. No positive results for (1) are true in any dimension for all locally integrable $f$; however the answer is yes if we restrict our attention to $f$ 's which are locally in an appropriate $L^{p}$ class and $n \geqslant 3$. Specifically, we have Theorem A.

An expanded version of an invited address presented by Professor Wainger to a meeting of the American Mathematical Society in Urbana, Illinois on March 19, 1976; received by the editors October 3, 1977.

AMS (MOS) subject classifications (1970). Primary 42A68, 28A15, 44A25.

'Both authors were supported by NSF grants.

2 References to some other recent results of a different nature will be found in part III. 
TheOREM A. If $n \geqslant 3$ and $f$ is locally in $L^{p}, p>n /(n-1)$,

$$
\lim _{\varepsilon \rightarrow 0} \int f(x-\varepsilon y) d \sigma(y)=f(x) \text { a.e. }
$$

This is false if $p \leqslant n /(n-1)$, for any $n$.

We do not know what happens if $n=2$ and $p>2$. It is to be understood that part of the assertion of Theorem $A$ is that the limit above is well defined for almost every $x$.

Incidentally, the analogue of Theorem A with the sphere replaced by the boundary of a cube is false. This should be the first hint to the reader that curvature will play a decisive role in these matters.

For our second question we let $\gamma(t)$ be a continuous curve in $R^{n}$ with $\gamma(0)=0$. We ask, does

$$
\lim _{h \rightarrow 0} \frac{1}{h} \int_{0}^{h} f(x-\gamma(t)) d t=f(x) \quad \text { a.e.? }
$$

The answer to (2) is yes if $\gamma(t)$ is a half-line, and this is, in fact, an easy consequence of the one-dimensional theory. We shall (in Part III) give examples of $C^{\infty}$ curves $\gamma(t)$ such that the answer to (2) is negative even if we restrict ourselves to the class of bounded functions. Thus to obtain positive answers to question (2) it is necessary to restrict attention to a subclass of curves. Again curvature is crucial. We obtain positive results if $\gamma$ has an appropriate amount of curvature. Let us say that a $C^{\infty}$ curve $\gamma(t)$ in $R^{n}$ is well-curved if $\gamma(0)=0$ and a segment of the curve containing the origin lies in the subspace of $R^{n}$ spanned by

$$
\left.\frac{d^{j}}{d t^{j}} \gamma(t)\right|_{t=0}, \quad j=1,2,3, \ldots{ }^{3}
$$

The following result holds:

THEOREM B. Let $f$ be locally in $L^{p}, p>1$. Then

$$
\lim _{h \rightarrow 0} \frac{1}{h} \int_{0}^{h} f(x-\gamma(t)) d t=f(x) \text { a.e. }
$$

provided $\gamma(t)$ is well-curved.

To see the full significance of Theorems A and B, we shall, in \$2, review relevant classical aspects of singular integral theory, differentiation theory, and especially the relation between them. This will suggest the control of appropriate maximal functions is the basic underlying analytical problem, and that there should be singular integral operators related to the curves $\gamma(t)$. These operators are defined by

$$
\mathcal{H} f(x)=\int_{-1}^{1} f(x-\gamma(t)) \frac{d t}{t} .
$$

( $F$ is well defined as a principal value integral if $f$ is smooth.)

${ }^{3}$ Note that in $R^{2}$ if $\gamma$ has nonvanishing curvature at the origin, it is well-curved. Similarly in $R^{3}$, if it has nonvanishing curvature and nonvanishing torsion. Also any real-analytic curve in $R^{n}$ is well-curved. 
In fact, our subject matter actually arose with the study of $\mathcal{H}$ rather than differentiation theorems, because results on $\mathcal{H}$ can be thought of as building blocks for large classes of singular integral operators.

In view of this, our second main goal will be to prove inequalities of the type

$$
\|\mathcal{H} f\|_{p} \leqslant A_{p}\|f\|_{p}, \quad 1<p<\infty .
$$

We shall show

THEOREM C.

$$
\|\mathcal{H} f\|_{p} \leqslant A_{p}\|f\|_{p}, \quad 1<p<\infty,
$$

provided $\gamma$ is well-curved.

This assertion is false for arbitrary $C^{\infty}$ curves.

We shall begin in $\$ 2$, as we have indicated, with a review of some of the classical ideas of maximal functions, differentiation theorems, singular integrals, their relationships and their applications. We shall then try to indicate the difficulty of carrying over the old methods to the present problems. Finally we shall try to explain new ideas that enter our analysis; namely, we show how to exploit the curvature of the curves and spheres mentioned above-via the Fourier transform-to obtain Theorems A, B, and C above as well as other related results.

Part II will be more technical and will contain the detailed proofs of the new results described in the first part. The final part will be a brief epilogue-where further results will be stated and some problems will be posed.

We want to take this opportunity to record our great debt to Alexander Nagel and Nestor Riviere with whom we have worked side-by-side on several of the results described below. If we have shared some disappointments, we have also shared the satisfaction of the fruits of this labor.

2. Background. We shall begin by reviewing those parts of the theory of harmonic analysis which will be directly relevant to us. We focus our attention on two kinds of basic estimates made in the theory of singular integrals: $L^{2}$ estimates, and $L^{p}$ estimates. For the former the crucial tool is the Fourier transform, as is well known; the latter proceeds by using ideas which have a very different origin, namely covering lemmas leading to the "maximal functions" of Hardy-Littlewood or differentiation theorems to control singular integral operators. We shall anticipate one of our main ideas when we say that, in distinction to what had been done in the past, we shall use singular integrals, or more precisely variants of Littlewood-Paley functions to help estimate maximal functions. Thus we shall control maximal functions at least in part by the use of the Fourier transform, and in effect use the Fourier transform to prove differentiation theorems.

2.a $L^{2}$ estimates and the Fourier transform. There is, to begin with, the Plancherel theorem. For $f$ an appropriate function on $R^{n}$, its Fourier transform $\hat{f}$ is given by

$$
\hat{f}(\xi)=\int_{R^{n}} \exp (2 \pi i x \cdot \xi) f(x) d x
$$


The mapping $f \rightarrow \hat{f}$ extends to a unitary operator on $L^{2}\left(R^{n}\right)$; thus in particular,

$$
\|\hat{f}\|_{2}=\|f\|_{2} \text {. }
$$

The rather immediate application of Plancherel's theorem is then as follows. Consider any operator $T$ of convolution type

$$
T: f \rightarrow \int K(y) f(x-y) d y .
$$

In order not to get entangled in technicalities we assume here that the kernel $K$ is quite general (a tempered distribution) and that $T$ is thus initially defined for $f$ which belong to the test class $\delta$ of Schwartz. (5) is the most general operator (defined on $\delta$ ), which commutes with translations. Now the condition that $T$ extends to a bounded operator on $L^{2}\left(R^{n}\right)$ (to itself) is a very simple one: Namely $\hat{K}$ (the Fourier transform of $K$ as a distribution) should be equivalent to a bounded function. Let us give several examples of this. The first is the Hilbert transform $H$ on $R^{1}$. Here $K$ is the principal-value distribution representing the function $K(x)=1 / \pi x$. A simple argument (in effect, an evaluation of a classical integral) shows that $\hat{K}(\xi)=i$ sgn $\xi$. These considerations show that the Hilbert transform is bounded on $L^{2}$ (and in fact unitary). Among the many reasons why the Hilbert transform $H$ is fundamental in harmonic analysis on $R^{1}$ we list the following:

(a) $H$ connects the real and imaginary parts of functions on $R^{1}$ which are boundary restrictions of suitable holomorphic functions in the upper-half plane. Closely connected with this is the fact that the operator $C=(I+$ $i H) / 2$ is the orthogonal projection on $L^{2}\left(R^{1}\right)$ whose range is the restrictions of holomorphic functions (more precisely those belonging to the Hardy space $\left.H^{2}\left(R^{1}\right)\right)$.

(b) $H$ is characterized (up to a constant multiple), by very simple structural properties. $H$ commutes with translations and also with dilations (the mappings $f(x) \rightarrow f(t x), t>0$ ), and anticommutes with the reflection $f(x) \rightarrow$ $f(-x)$. These facts are, on the formal level, simple consequences of the corresponding homogeneity of the kernel $1 /(\pi x)$ (or the multiplier $i$ sgn $\xi$ ).

To discuss examples for $R^{n}, n>1$, we turn to partial differential equations, and in particular to constant coefficient elliptic differential operators. A differential operator $P(\partial / \partial x)=\Sigma_{|\alpha| \leqslant k} a_{\alpha}(\partial / \partial x)^{\alpha}$, with $(\partial / \partial x)^{\alpha}=$ $\left(\partial / \partial x_{1}\right)^{\alpha_{1}} \ldots\left(\partial / \partial x_{n}\right)^{\alpha_{n}}, \alpha=\left(\alpha_{1}, \ldots, \alpha_{n}\right),|\alpha|=\alpha_{1}+\cdots+\alpha_{n}$ or order $k$ is said to be elliptic if the part of the characteristic polynomial of degree $k$, $\Sigma_{|\alpha|=k} a_{\alpha} \xi^{\alpha}$, vanishes only when $\xi=0$. For these operators using the Fourier transform one can prove the a priori estimate

$$
\left\|\left(\frac{\partial}{\partial x}\right)^{\alpha} u\right\|_{2}<A\left\{\left\|P\left(\frac{\partial}{\partial x}\right) u\right\|_{2}\right\}, \quad|\alpha|<k,
$$

for $u$ which are suitably smooth.

This estimate, and others of the same kind, can be used as the starting point for proving existence and regularity results for elliptic differential operators with constant coefficients, and then one can prove as a consequence similar estimates and results for equations with variable 
coefficients. Included among these estimates are the "Gårding inequalities". For further discussion of these matters see e.g. Yosida [1965, Chapter VI], and Trèves [1975, Chapter III].

Let us look at (6) more carefully. We shall get more insight into the situation if we consider the corresponding inequalities which are homogeneous under the standard dilations (the dilations map $u$ to $u_{t}$, where $u_{t}(x)=t^{-n} u\left(\delta_{t}(x)\right), \delta_{t}(x)=\left(t x_{1}, \ldots, t x_{n}\right)$ with $x=\left(x_{1}, \ldots, x_{n}\right)$ for all $t>$ $0)$. We then restrict our attention to homogeneous elliptic operators, i.e.

$$
P\left(\frac{\partial}{\partial x}\right)=\sum_{|\alpha|=k} a_{\alpha}\left(\frac{\partial}{\partial x}\right)^{\alpha} \text {. }
$$

One obtains as a variant of (6),

$$
\left\|\left(\frac{\partial}{\partial x}\right)^{\alpha} u\right\|_{2}<A\left\|P\left(\frac{\partial}{\partial x}\right) u\right\|_{2}, \quad|\alpha|=k,
$$

whenever e.g. $u$ is smooth and has compact support. It turns out the inequality (7) essentially contains the full force of (6).

Now there is a (unique) bounded operator $T$ on $L^{2}$, which maps $P(\partial / \partial x) u$ to $(\partial / \partial x)^{\alpha} u$ (for each $P$ and each $\alpha$, with $|a|=k$ ), i.e.

$$
\left(\frac{\partial}{\partial x}\right)^{\alpha}=T P\left(\frac{\partial}{\partial x}\right)
$$

This operator $T$ can be written as $T=T_{0}+c I$, and when $c$ is suitably chosen, the operator $T_{0}$ is of the form (5) where the kernel $K$ is a principal-value distribution, represented by a function $K(x), x \neq 0$, which satisfies the following properties

$$
\begin{aligned}
& \text { (a) } K \text { is homogeneous of degree }-n \text {, i.e. } \\
& K\left(\delta_{t}(x)\right)=t^{-n} K(x), t>0, \\
& \text { (b) } K \text { is } C^{\infty} \text { away from the origin, } \\
& \text { (c) } \int_{|x|=1} K(x) d \sigma(x)=0 .
\end{aligned}
$$

In fact (a) is the reflection of the equal homogeneity of $P(\partial / \partial x)$ and $(\partial / \partial x)^{\alpha}$. Property (b) is closely related to the regularity of $P$, namely any solution of $P(\partial / \partial x) u=0$ is $C^{\infty}$ where defined. (c) is a consequence of the subtraction of the appropriate constant multiple of the identity in defining $T_{0}$.

Now we come to an important turning point. One can take properties (9) (a), (b), and (c) as the starting point (in fact (b) can be considerably relaxed), and study the class of operators $T$ given by (5) with $K$ satisfying (9) (a), (b), and (c). These are the operators of Mihlin [1950], and Calderón and Zygmund [1952].

What are the operators we get in this way? When $n=1$ there is (up to a constant multiple) only one $K$ which satisfies (9) (a) and (c) ((b) is redundant when $n=1$ ), and this is $K(x)=1 / \pi x$, so we are back to the Hilbert transform. However when $n>1$, there are infinitely many examples and variants, some not directly connected with partial differential equations, but of interest in other problems in analysis. For all $K$ of type (9) (a)-(c) one can prove 


$$
\|T f\|_{2} \leqslant A\|f\|_{2}
$$

and this follows once we know that $\hat{K}$ is a bounded function. For further discussion see e.g. Stein [1970a, Chapters II and III].

We shall now turn to other types of estimates, the $L^{p}$ estimates, for operators such as $T$.

2(b) $L^{p}$ estimates and maximal functions. It may be useful if at this stage we try to answer a question that is often asked. Given an operator $T$, what is the interest in $L^{p}$ estimates for it? The answer is, first of all, that we usually wish to compare the size of $T f$ to the size of $f$, and inequalities involving $L^{p}$ norms are often a convenient way of making such comparisons. But there is an additional reason. When we are making $L^{p}$ estimates, we are often gaining a much more complete grasp of how the size of $T f$ is controlled by the size of $f$. We will see some of this in several examples below. This control will then be in terms of the "distribution function" of $T f$, that is $|\{x:|T f(x)|>\alpha\}|$, in terms of the corresponding objects which involve $f$, or in terms of certain special operators acting on $f$.

With these justifications taken as understood, we turn to some of the actual $L^{p}$ estimates. The source of many of these must be different from that of the special case $p=2$, since those used the Plancherel theorem, and of course there is no analogue of this theorem for other values of $p$. A basic tool here is the maximal function $M$, which represents the maximum of averages of $|f|$ taken over balls. More precisely, we write whenever $f$ is locally integrable,

$$
M f(x)=\sup _{r>0} \frac{1}{c r^{n}} \int_{|y| \leqslant r}|f(x-y)| d y
$$

where $c$ is the volume of the unit ball. Since

$$
\lim _{r \rightarrow 0} \frac{1}{c r^{n}} \int_{|y|<r} f(x-y) d y=f(x) \text { a.e. }
$$

it is clear that $M f$ dominates $f$. It turns out that $M f$ is in fact not much larger than $f$ (in a suitable sense), but has many advantages over $f$; in particular $M f$ is the crucial basic operator that provides a large amount of the control of $T f$ for operators $T$ satisfying 9 (a), (b), (c).

Not only is $M$ basic in singular integral theory, but also appropriate control of $M$ is equivalent to Lebesgue's differentiation theorem. This, then, is the promised tie between differentiation theorems and singular integrals.

The maximal function $M$ also plays a fundamental role in studying other "approximations to the identity" which are very useful in applications. Let $\Phi$ be a fixed nonnegative function which is radial and nonincreasing, and so that $\int_{R^{n}} \Phi(x) d x=1$. Set $\Phi_{\varepsilon}(x)=\varepsilon^{-n} \Phi(x / \varepsilon)$. Then whenever $f \in L^{p}\left(R^{n}\right)$, $1 \leqslant p \leqslant \infty,\left(f * \Phi_{\varepsilon}\right)(x) \rightarrow f(x)$ a.e. as $\varepsilon \rightarrow 0$. The connection with the maximal function is that

$$
\sup _{\varepsilon}\left|f * \Phi_{\varepsilon}(x)\right| \leqslant M f(x) .
$$

We shall enter into these applications of $M$ in more detail in a moment. First, however, we shall consider the basic properties of $M$.

If $f \in L^{p}\left(R^{n}\right), 1<p \leqslant \infty$, then $M f \in L^{p}$, and 


$$
\|M(f)\|_{p} \leqslant A_{p}\|f\|_{p} .
$$

For $p=1$ this kind of estimate cannot hold, but there is a corresponding result (the "weak type $(1,1)$ " inequality)

$$
|\{x \mid M f(x)>\alpha\}| \leqslant A\|f\|_{1} / \alpha, \quad \alpha>0,
$$

and this inequality is in short the key to the whole theory of $M$. (The fundamental facts about the maximal function when $n=1$ were discovered by Hardy and Littlewood [1930]. The $n$-dimensional theory is due to Wiener [1939], and Marcinkiewicz and Zygmund [1939]. That (13) implies Lebesgue's theorem is elementary. The fact that (13) is equivalent to Lebesgue's theorem is less simple. It follows from Stein [1961]. For material on other approximations of the identity see Stein and Weiss [1971, pp. 13 and 59].)

We shall say a few words about the proof of (13). It follows directly from a covering lemma of Vitali type, which by now has many variants, and a trivial remark. The trivial remark is

(14a) If $M f(x)>\alpha, x$ is contained in some ball $B$ centered at $x$ with

$$
\frac{1}{\alpha} \int_{B} f \geqslant|B| \text {. }
$$

We state a particularly simple special case of the covering lemma:

(14b) BASIC LEMMA. Suppose $E$ is a measurable set in $R^{n}$, which is contained in the union of finitely many balls, $B_{1}, B_{2}, \ldots, B_{N}$. Then there is a subcollection of these balls $B_{i_{1}}, B_{i_{2}}, \ldots, B_{i_{n}}$, whose interiors are disjoint, so that

$$
\sum|B| \geqslant C|E|
$$

( $C$ is a positive constant depending only on the dimension $n$ ).

The key to the Basic Lemma, is the following

(15) GEOMETRIC FACT. If $B_{1}$ and $B_{2}$ are two balls with nonempty intersection, and diam $B_{1} \geqslant \frac{1}{2} \operatorname{diam} B_{2}$, then

$$
B_{1}^{*} \supset B_{2}
$$

where $B_{1}^{*}$ is the sphere with the same center as $B_{1}$ and diameter 5 times that of $B_{1}$.

The demonstration that $(15) \rightarrow(14) \rightarrow(13) \rightarrow(12)$ can be found in many places, e.g. in Stein [1970a, Chapter I].

To see that (13) implies (11), note first that (11) is obvious if $f$ is continuous. Next, since the result is local in nature we may assume that $f$ is integrable over all of $R^{n}$, and approximate it in $L^{1}$ norm by continuous functions. Finally (13) allows us to control the error when we replace $f$ by these continuous functions.

We turn now to the operator $T$ of (5), discussed before, with $K$ satisfying the condition (9). We then have the basic application of the maximal function $M$ to $L^{p}$ inequalities.

THEOREM. $f \rightarrow T(f)$ extends to a bounded operator on $L^{p}\left(R^{n}\right), 1<p<\infty$.

We shall not sketch the proof of this theorem, but we shall content ourselves with the following observations. What is actually proved (from 
which the theorem follows without too much difficulty) is an estimate for the distribution function of $T f$,

$$
|\{x|T f(x)|>\alpha\}|<A|\{x \mid M f(x)>\alpha\}|+\frac{B}{\alpha^{2}} \int_{|f|<\alpha}|f|^{2} d x .
$$

The first term of the right side of (16) gives the control in terms of the maximal function, and the second term comes from the $L^{2}$ estimate made earlier. (See Calderón and Zygmund [1952], also Stein [1970a, Chapter II].) With some additional arguments one can remove (at least in appearance) the $L^{2}$ term, and state the estimate as a conditional inequality in terms of the maximal function $M$. Namely for every $\gamma>0$ (with $\gamma$ taken to be small) there is a positive constant $A_{\gamma}$, so that for all $\alpha>0$

$$
|\{x|| \bar{T} f(x) \mid>\alpha\}|<\left|\left\{x \mid A_{\gamma} M f(x)>\alpha\right\}\right|+\gamma|\{x|| \bar{T} f(x) \mid>\alpha / 2\}|^{4}
$$

(For estimates of this kind see Burkholder and Gundy [1970], and Coifman [1972].)

3. Spherical maximal function. Just as Lebesgue's differentiation theorem is closely tied to $M f$, the differentiation theorems described in the preface are consequences of estimates for appropriate maximal functions. We consider first the maximal function appropriate for Theorem A.

We consider here averages not over balls, but over spheres. That is we consider

$$
M_{t} f(x)=\int_{|y|=1} f(x-t y) d \sigma(y), \quad t>0,
$$

where $d \sigma$ is the rotationally invariant measure on the unit sphere with total mass 1 . We define

$$
\mathfrak{N} f(x)=\sup _{t>0}\left|M_{t} f(x)\right|
$$

and ask whether there is an estimate of the form

$$
\|\Re f\|_{p} \leqslant A_{p}\|f\|_{p} \text { for some } p, 1<p<\infty \text { ? }
$$

(To avoid technicalities with the definition of $\Re$, we assume $f$ is smooth. We shall show later that if (18) is true for some $p_{0}<\infty$ and smooth $f$, $\mathfrak{T}$ is well defined and (18) continues to hold for all $f$ in $L^{p_{0}}$.)

It is obvious that $M f(x)<(\Re(f))(x)$, if $f \geqslant 0$, where $M$ is the maximal function defined in terms of balls, so any result of the kind (18) would represent an extension of the classical maximal theorem. The obvious things that can be said about (18) are in the negative. First, (18) is false when $n=1$, for any $p<\infty$. To see this one merely needs to take an unbounded $f$ which belongs to every $L^{p}\left(R^{1}\right), p<\infty$. Next using the same idea (see Part III) one can see that when $n \geqslant 2$, then (18) is false as long as $p \leq n /(n-1)$. What is surprising is that the following result holds.

TheOREM $\mathrm{A}^{\prime}$. Suppose $n \geqslant 3$, and $p>n /(n-1)$. Then (18) is valid.

Thus when $n \geqslant 3$, we know exactly for which $p$ (18) can hold. For $n=1$

\footnotetext{
${ }^{4}$ Here $\bar{T}$ is the maximal operator corresponding to variable truncations of $T$.
} 
we also know the answer, trivially. Only the case $n=2, p>2$ still remains undecided.

Theorem $\mathbf{A}^{\prime}$ easily implies the positive assertions of Theorem $\mathbf{A}$ of the preface. The negative results of Theorem A follow in the same way.

Theorem $A^{\prime}$ and the method of its proof (which we shall sketch in $\$ 5$ below) has several consequences which we now mention. First there is the following paradoxical measure-theoretic assertion (which gets us back to the technicalities we mentioned before). Whenever $E$ is a Lebesgue measurable set in $R^{n}, n \geqslant 3$, then almost every $x \in R^{n}$ has the property that $E \cap\{y|| x$ $-y \mid=t\}$ is $(n-1$ dimensional) measurable, for every $t>0$. Moreover if $E$ has measure zero, then $E \cap\{y|| x-y \mid=t\}$ has (n-1 dimensional) measure zero, for every $t$, for almost every $x$. These curious considerations are the ones mentioned above which allow us to give precise meaning to Theorems $\mathrm{A}$ and $\mathrm{A}^{\prime}$ for general $f$.

The complete study of the maximal function $\mathfrak{T}$ requires us to consider together with the spherical averages $M_{t}$ also the averages $M_{t}^{\alpha}$, which when $\alpha>0$ are defined by

$$
M_{t}^{\alpha}(f)(x)=c(\alpha) \int_{|y|<t}\left(1-\frac{|y|^{2}}{t^{2}}\right)^{\alpha-1} f(x-y) d y,
$$

for appropriate $c(\alpha)$, and are given by analytic continuation in $\alpha$ for other $\alpha$ (see $\$ \$ 3$ and 7 in Part II). (For $\alpha=0$ we recover $M_{t}$.) Now the functions $M_{t}^{\alpha}$ have an independent interest-for special values of $\alpha$ they give us the solution of the wave equation

$$
\sum_{j=1}^{n} \frac{\partial^{2} u}{\partial x_{j}^{2}}=\frac{\partial^{2} u}{\partial t^{2}}
$$

satisfying $u(x, 0)=0, \partial u(x, 0) / \partial t=f(x)$. More precisely, for a suitable constant $c=c_{n}$, the solution of this problem can be written as

$$
u(x, t)=c t M_{t}^{\alpha}(f)(x)
$$

with $\alpha=3 / 2-n / 2$. When $n=1$ we have, of course, the familiar solution

$$
u(x, t)=\frac{1}{2} \int_{-t}^{t} f(x-s) d s .
$$

The classical differentiation theorem, namely

$$
\frac{1}{2 t} \int_{-t}^{t} f(x-s) d s \rightarrow f(x) \text { a.e., }
$$

can by the above be generalized as follows: $u(x, t) / t \rightarrow f(x)$ a.e. as $t \rightarrow 0$ when $f$ is locally in $L^{p}\left(R^{n}\right)$, with $p>2 n /(n+1)$. This is a consequence of the maximal theorem for $M_{t}^{\alpha}$ (Theorem 14 in Part II); see also Stein [1976a].

4. Maximal functions and Hilbert transforms along curves, and nonisotropic dilations. Let $\gamma(t)$ be a continuous curve with $\gamma(0)=0$. To prove Theorem B, we introduce an appropriate maximal function

\footnotetext{
${ }^{5}$ We are indebted to A. Cordoba for suggesting this kind of application.
} 


$$
\mathscr{T} f(x)=\sup _{0<h<1} \frac{1}{h} \int_{0}^{h}|f(x-\gamma(t))| d t .
$$

(This is, of course, not the same $\Re$ as occurs in \$3.) We shall show later, and this is not difficult, that $\mathfrak{N}$ is well defined for any locally integrable $f$.

We shall prove

THEOREM B'. If $\gamma$ is well-curved,

$$
\|\Re f\|_{p}<A_{p}\|f\|_{p}, \quad 1<p<\infty .
$$

Theorem B' implies Theorem B. If we followed the classical lines of $\$ 2$, we would try to prove Theorem $\mathbf{C}$ as a consequence of the theory of $\Re$. This will however not be the case.

An important feature of well-curved curves appears when we consider the simplest two dimensional examples; namely,

$$
\gamma(t)=\left(t^{j}, t^{k}\right), \quad t \geqslant 0,
$$

where $j$ and $k$ are distinct positive integers. These examples are not as special as they might seem at first sight, since the general well-curved $\gamma$ (in two dimensions) is in a sense approximated by these "model" curves. The new feature of the examples is that $\gamma(t)$ is homogeneous with respect to some nonisotropic dilations of the underlying $R^{2}$. That is we have a one parameter family of dilations, $\delta_{t}$, of $R^{2}$ to itself, for $t>0$, given by

$$
\delta_{t}(x)=\left(t^{j} x_{1}, t^{k} x_{2}\right), \quad t>0,
$$

and

$$
\gamma(t s)=\delta_{t} \gamma(s), \quad t>0, s>0 .
$$

In fact $\gamma(t), t \geq 0$, is actually the orbit of the point $(1,1)$ under these dilations, and our study of $\mathscr{N}$ and $\mathcal{H}$ in this case as well as the more general one will depend crucially on these dilations.

One can present the general notion of nonisotropic dilations as follows. It is a one-parameter family $\delta_{t}, t>0$, of linear transformations of $R^{n}$ with the properties

(a) $\delta_{t} \delta_{s}=\delta_{t s}, t, s>0$,

(b) $\delta_{1}=$ Identity,

(c) $\delta_{t}(x) \rightarrow 0$, as $t \rightarrow 0$, for any $x \in R^{n}$.

Consider a "homogeneous curve" defined by $\gamma(t)=\delta_{t}(e)$, for $t \geqslant 0$, and $\gamma(t)=\delta_{-t}(f)$, for $t \leqslant 0$, where $e$ and $f$ are two vectors in $R^{n}$. It will be necessary to assume that the two pieces, the one for positive $t$ and the one for negative $t$ are compatible in the following sense: the linear space spanned by $\{\gamma(t)\}_{t>0}$ equals the linear space spanned by $\{\gamma(t)\}_{t<0}$. (Without this condition there could be no theory for $\mathcal{H}$.) However for $\mathscr{N}$ only $\gamma(t), t>0$, is relevant.

THEOREM D. The inequalities

$$
\|\Re f\|_{p}<A_{p}\|f\|_{p}, \quad 1<p<\infty
$$

and

$$
\|\mathcal{H} f\|_{p}<A_{p}\|f\|_{p}, \quad 1<p<\infty,
$$


hold for the class of curves described above.

It should be stressed that Theorem $B^{\prime}$ does not imply (20) or vice versa, (similarly with Theorem $\mathrm{C}$ and 21 ). However all these results have common roots in that both depend in a key way on the use of nonisotropic dilations. These dilations are given, in effect, explicitly for homogeneous curves, but are implicit when $\gamma$ is well-curved.

The nonisotropic dilations which are so important for us occur also in significant ways in several other questions in analysis. We mention briefly the following examples:

(1) Estimates for solutions of constant coefficient parabolic differential operators. (See Jones [1964], Fabes [1966], Fabes and Riviere [1966] and [1967].) The simplest instance of this occurs when one considers the operator of the heat equation,

$$
L(u)=\frac{\partial u}{\partial x_{1}}-\sum_{j=2}^{n} \frac{\partial^{2} u}{\partial x_{j}^{2}} .
$$

If one wishes to have analogues of (6), (7) and their $L^{p}$ versions, then one needs to consider singular integral operators with kernels satisfying conditions akin to (9) however (9) (a) should be replaced by the condition

$$
K\left(\delta_{t}(x)\right)=\left(\operatorname{det} \delta_{t}\right)^{-1} K(x), \quad t>0,
$$

where $\delta_{t}(x)=\left(t^{2} x_{1}, t x_{2}, \ldots, t x_{n}\right)$.

The Hilbert transform $\mathcal{K}$ for homogeneous curves corresponding to these dilations may be viewed as the basic building block for this class of singular integrals, if one adapts suitably the "method of rotations" of Calderón and Zygmund [1956].

(2) Estimates for some subelliptic partial differential equations. One can go further in this direction and study some general classes of variable coefficient subelliptic partial differential equations. Without going into detail we can say that in this context $R^{n}$ has its additive structure replaced by another group multiplication and the dilations in question are required to be automorphisms of the group structure. The analogues of the operators (5) (with the appropriate variants of (9)) are then convolution operators with respect to the group structure. A theory along these lines has been carried out, and gives the appropriate class of singular integral operators, sharp estimates, etc. for the second-order hypoelliptic operators of Hormänder [1967] type. (See Rothschild and Stein [1976].) A particularly interesting example arises in the setting of the inhomogeneous Cauchy-Riemann equations for domains (and boundaries) in several complex variables. Here we consider $R^{2 n+1}$ as the Heisenberg group; that is $R^{2 n+1}=C^{n} \times R^{1}=\left\{(z, t), z \in C^{n}, t \in R\right\}$, with the group multiplication $(z, t) \cdot\left(z^{\prime}, t^{\prime}\right)=\left(z+z^{\prime}, t+t^{\prime}+2 \operatorname{Im} z \cdot \bar{z}^{\prime}\right)$. Observe that the dilations $\delta_{s}(z, t)=\left(s z, s^{2} t\right), s>0$, are compatible with the group multiplication; while the standard ("isotropic") dilations are not, and hence are largely irrelevant. For further details see Folland and Stein [1974].

(3) Analysis on symmetric spaces. Various problems related to a semisimple Lie group $G$, or its symmetric space $G / K$ lead to such nonisotropic dilations. We mention here only the extensions of Fatou's theorem dealing with 
existence almost everywhere of boundary values of harmonic functions (Poisson integrals) on $G / K$. See the surveys of Stein [1971], and Koranyi [1972], and the recent results in Stein [1976c].

5. Sketch of the proofs of the main theorems. Let us begin by pointing out some of the difficulties of carrying out the program of $\$ 2$ for curves $\gamma(t)$ in $R^{2}$. We make matters a little clearer by fattening out the curves slightly. Then we wish to consider sets

$$
E_{\varepsilon, \delta}=\{(x, y) \mid 0<x<\varepsilon, \gamma(x)<y<\gamma(x)+\delta\} .
$$

Then to obtain the analogue to the inequality (13), we would like to prove (14b) (with the balls $B_{\varepsilon}$ replaced by $E_{\varepsilon, \delta}$ and $C$ independent of $\delta$ ). The difficulty is that the analogue of (15) is miserably false. (Actually we do not know if the analogue of (13) is true.) Even then we don't know anything like (16) so that we can't use results for $\mathfrak{T}$ to obtain results about $\mathcal{H}$.

In the case of spherical averages, similar comments can be made, but the situation is much worse. The analogue of (13) is false, and in addition the analogue of $(14 a)$ is no longer valid.

So much for the difficulties. We turn now to the outline and main ideas behind the proofs of Theorems $A^{\prime}, B^{\prime}, C$, and $D$.

The estimates for maximal functions related to spheres and curves are based on the same device: A Tauberian argument effectuated by a variant of the Littlewood-Paley $g$-function. Recall that one of the classical Tauberian theorems can be stated as follows. Suppose $\Sigma a_{k}$ is a numerical series, and we are interested in its convergence i.e. the question whether

$$
S_{n}=\sum_{k=1}^{n} a_{k} \rightarrow l \text { as } n \rightarrow \infty \text {. }
$$

Then a sufficient condition for this to hold is that the averages tend to $l$, i.e.

$$
\frac{1}{n} \sum_{k=1}^{n} S_{k} \rightarrow l,
$$

together with the condition on the coefficients,

$$
a_{k}=O(1 / k) \text { as } k \rightarrow \infty .
$$

There are many variants of (22) that abound in the literature. For us, what is relevant is that instead of (22) it suffices to have

$$
\sum_{k=1}^{\infty} k\left|a_{k}\right|^{2}<\infty
$$

(For a general treatment of Tauberian theorems see Hardy [1949], and in particular Theorem 69.)

The quadratic nature of (23) provides the link with $L^{2}$ theory and thus the Fourier transform.

Let us now describe the proof of Theorem $\mathbf{A}^{\prime}$ in the special case when $p=2$ and $n \geqslant 4$, where the argument is particularly simple. We are interested, in effect, with $\lim _{t \rightarrow 0} M_{t} f$, but we cannot control the averages of $M_{t} f$ in terms of the usual maximal function $M$. So we seek a Tauberian condition 
akin to (23). What we need is expressed by the operator

$$
g(f)(x)=\left(\int_{0}^{\infty}\left|\frac{d}{d t} M_{t}(f)(x)\right|^{2} t d t\right)^{1 / 2} .
$$

(We are interested at this stage in a priori inequalities, so we may assume $f$ to be smooth, and thus (24) is well defined.)

The main point about $g(f)$-the Tauberian condition-is that

$$
\|g(f)\|_{2}<C\|f\|_{2} \text { if } n>4 \text {. }
$$

Let us assume (25) momentarily. Now

$$
t^{n} M_{t}=\int_{0}^{t} \frac{d}{d s}\left(M_{s} s^{n}\right) d s,
$$

so

$$
\begin{aligned}
M_{t}(f)(x)= & t^{-n} n \int_{0}^{t} M_{s} f(x) s^{n-1} d s \\
& +t^{-n} \int_{0}^{t} s^{n} \frac{d}{d s}\left(M_{s} f(x)\right) d s .
\end{aligned}
$$

The first term is obviously controlled by $M(f)(x)$ of $\$ 2$, and the second term can be estimated by

$$
\left(\int_{0}^{t} s\left|\frac{d M_{s}}{d s}\right|^{2} d s\right)^{1 / 2} t^{-n}\left(\int_{0}^{t} s^{2 n-1} d s\right)^{1 / 2}<c g(f)(x) .
$$

Therefore,

$$
\Re f(x)<c\{M f(x)+g(f)(x)\},
$$

and (25) together with (12) proves that

$$
\|\Re f\|_{2}<A\|f\|_{2} \text {, if } n>4 \text {. }
$$

We return to (25). As is well known

$$
\left(M_{\downarrow} f\right)^{\wedge}(\xi)=m(t|\xi|) \hat{f}(\xi),
$$

where

$$
m(u)=c u^{-n / 2+1} J_{n / 2-1}(2 \pi u), \quad u>0 .^{6}
$$

Thus by the Plancherel formula (25) is equivalent to the inequality

$$
\int_{0}^{\infty}\left|\frac{d m(t)}{d t}\right|^{2} t d t=c^{2}<\infty .
$$

The integral in (26) converges since $m^{\prime}$ (as well as $m$ ), satisfies the inequality

$$
m^{\prime}(t)=O\left(t^{-n / 2+1 / 2}\right)
$$

as $t \rightarrow \infty$, while $m$ is smooth locally.

\footnotetext{
${ }^{6}$ See e.g. the closely related formulae in Stein and Weiss [1971, p. 155].
} 
The estimate (27), which makes the $L^{2}$ theory possible, is a direct reflection of the curvature of the sphere, since of course $m$ is essentially the Fourier transform of the uniform measure on the unit sphere. The observation connecting the decay of $m$ and the curvature of the sphere is highlighted by similar known estimates where the sphere is replaced by various generalizations (e.g. smooth compact hypersurfaces with strictly positive Gaussian curvature). For these facts and generalizations see Hlawka [1950], Herz [1962a], Littman [1963], and Randol [1972]. In the other direction, if all curvature assumptions are dropped then the best we can hope for instead of (27) is $m^{\prime}(t)=O(1)$, and then of course the rest of our argument breaks down.

Before we continue it may be of interest to make some remarks of a historical nature concerning the role of $g$-functions. Littlewood and Paley [1931], [1936], [1937] introduced the expression

$$
\left\{\int_{0}^{1}(1-r)\left|F^{\prime}\left(r e^{i \theta}\right)\right|^{2} d r\right\}^{1 / 2},
$$

where $F$ is holomorphic in the unit disc, and used it in crucial ways to prove $L^{p}$ inequalities for Fourier series. Since then many variants have been studied, including the Lusin "area integral", $g_{\lambda}^{*}$ functions, generalizations to $R^{n}$ etc. These extensions may be viewed as operators defined in terms of the Poisson integral of $f$ (or other approximations to the identity), where $f$ is a suitable function on $R^{n}$. These play a key role in multiplier theorems and $H^{p}$ theory for $R^{n}$. (See Zygmund [1959, Chapter 15], Stein [1970a], and Fefferman and Stein [1972].) Their importance in this connection is due to the fact that, roughly speaking, the norm of $g(f)$ is equivalent with the norm of $f$, while $g$ is more flexible than $f$ is under various transformations. Thus the $g$-functions become, in some sense, the central objects of study.

In the present context we have still other variants of these $g$-functions but here the role is changed from a primary one to a supporting one, in terms of Tauberian conditions. It should be emphasized that using variants of the $g$-functions in their Tauberian capacity is not without precedent. For example, in the general $L^{2}$ theory of orthogonal series, as in Kaczmarz and Steinhaus [1951, Chapter 5]; in the summability theory of multiple Fourier series and integrals (see e.g. Stein and Weiss [1971, Chapter 7], and in the theory of general symmetric diffusion and semigroups, as in Stein [1970b]).

We return to the proofs of our theorems. The arguments for the maximal inequalities for smooth curves and homogeneous curves, Theorem $B^{\prime}$ and (20) of Theorem $\mathrm{D}$, for $p=2$, are carried out in a similar vein as sketched above, and require the introduction of $g$-functions suitable to these problems. We describe the situation briefly. Suppose $\gamma(t)$ is a homogeneous curve, and let us make the additional assumption that $\{\gamma(t)\}_{t>0}$ spans all of $R^{n}$. Then one can show that

$$
\int_{a}^{b} \exp (i \xi \cdot \gamma(t)) d t=O\left(|\xi|^{-\sigma}\right), \quad \text { as } \xi \rightarrow \infty
$$

for some positive $\sigma$, and the estimate is uniform in $a$ and $b$ as long as they lie 
in a compact subinterval of $(0, \infty)$. This can be proved by using estimates of the van der Corput type. Now (28) is the substitute for (27) in proving $L^{2}$ boundedness of the appropriate $g$-functions, and hence the maximal function associated to the curve $\gamma$.

Instead of now describing how the $L^{p}$ inequalities are proved for $\Re$ it will be best to sketch first the proof of the $L^{p}$ inequalities for the Hilbert transform $\mathcal{H C}$ associated to a homogeneous curve $\gamma$.

We are dealing essentially with the operator formally defined by

$$
\mathcal{H} f(x)=\int_{-\infty}^{\infty} f(x-\gamma(t)) \frac{d t}{t}
$$

Its $L^{2}$ boundedness is equivalent with the boundedness of the multiplier, namely

$$
m(\xi)=\int_{-\infty}^{\infty} e^{i \xi \cdot \gamma(t)} \frac{d t}{t}
$$

where the nonabsolutely convergent integral is taken in the principal-value sense. Thus one first proves the boundedness of the function $m$. This turns out to be an easy consequence of the estimate (28).

In order to do the $L^{p}$ theory one needs a stronger version of this result. One lets $\delta_{t}^{*}$ be the adjoints of $\delta_{t}$ associated to $\gamma$. The $\delta_{t}^{*}$ form another one parameter family of dilations. Then we can construct an associated "norm function" $\rho^{*}$ with the following properties: $\rho^{*}(\xi)>0$, except when $\xi=0 ; \rho^{*}$ is $C^{\infty}$ away from the origin; and $\rho^{*}$ is homogeneous in the sense that $\rho^{*}\left(\delta_{t}^{*}(\xi)\right)=t \rho^{*}(\xi), t>0$. We then consider the fractional differentiation operator $D^{2}$, of complex order $z$, defined formally by

$$
\left(D^{z} f\right)^{\wedge}(\xi)=\left(\rho^{*}(\xi)\right)^{z} \hat{f}(\xi)
$$

Also one considers the analytic family of operators defined by

$$
T_{z}(f)=D^{z} \circ \int_{-\infty}^{\infty} f(x-\gamma(t))|t|^{2} \frac{d t}{t} .
$$

Note that $T_{0} f=\mathcal{H} f$.

Because of (28) one can prove

$$
\left\|T_{z} f\right\|_{2} \leqslant A_{z}\|f\|_{2}, \quad 0 \leqslant R(z)<\sigma .
$$

This result is stronger than the $L^{2}$ boundedness of $H=T_{0}$, since $T_{z}$ can be viewed as a convolution with a distribution more singular than that defining $H$. But the advantage of considering $T_{z}$ is that when $R(z)<0, T_{z}$ is now closer to a more traditional singular integral, in fact one for which by and large the methods of $\$ 2$ apply. Thus one can show that

$$
\left\|T_{z} f\right\|_{p}<A(z, p)\|f\|_{p}, \quad 1<p<\infty, R(z)<0 .
$$

A combination of (32) with (31) by analytic interpolation of operators gives the desired $L^{p}$ estimates for $H$.

The $L^{p}$ inequalities for the maximal function $M$ are proved in the same spirit. First the $L^{2}$ result described above can be refined, and the final result is obtained by complex interpolation involving the improved $L^{2}$ results and other $L^{p}$ results of a more standard nature. 
As we close this survey it may be worthwhile to see the patterns of the arguments retrospectively, in terms of the order in which the proofs were actually developed. The $L^{2}$ boundedness of $\mathcal{H}$ in the two-dimensional case $\gamma(t)=\left(t^{\alpha}, t^{\beta}\right)$ was proved by Fabes [1966]. He showed $m$ was bounded by use of complex integration. Then Stein and Wainger [1970] proved the $L^{2}$-boundedness for $\mathcal{H C}$ for homogeneous curves by using van der Corput's estimates for trigonometric integrals to obtain the boundedness of $m$. The first main breakthrough was the proof of the $L^{p}$ inequalities for $\mathcal{H}$, in a series of papers of Nagel, Riviere, and Wainger [1974] and [1976a] by a method akin to (but somewhat more indirect than) the one we have sketched. The next idea was the proof of the maximal theorem for the curve $\left(t, t^{2}\right)$ by Nagel, Rivière, and Wainger [1976b]. The argument was special to that situation but had the virtue of showing the usefulness of the Fourier transform in the proof of maximal inequalities. There followed a pair of papers (Stein [1976a], [1976b]) introducing the technique of $g$-functions in this problem and proving Theorem $\mathbf{A}^{\prime}$ and the maximal theorem for homogeneous curves. The chain of arguments was completed in Stein and Wainger [1976], which contains Theorem $B^{\prime}$ for $\mathfrak{N}$, the maximal theorem for smooth curves.

\section{PART II-SOME DETAILED PROOFS}

1. Dilations. By a one parameter group of dilations on $R^{n}$, we mean a collection of linear transformations $\delta_{t}, \delta_{t}: R^{n} \rightarrow R^{n}$ one for each $t>0$ having the following properties:

$(1-1) \delta_{s t}=\delta_{s} \delta_{t}$ and $\delta_{1}=$ identity,

$(1-2) \delta_{t} x \rightarrow 0$ as $t \rightarrow 0$ for every $x$ in $R^{n}$,

$(1-3) \delta_{t} x$ is jointly continuous in $t$ and $x$. (Actually (1-3) is a consequence of (1-1) and (1-2).)

Corresponding to the one parameter group of dilations $\delta_{t}$ we define a transformation on functions or distributions $\phi \rightarrow \phi_{t}$ where

$(1-4) \phi_{t}(x)=\left(\operatorname{det} \delta_{t}\right)^{-1} \phi\left(\delta_{t}^{-1} x\right)$. if

We shall also say that a curve $\gamma(t), t>0$, is homogeneous with respect to $\delta_{t}$

$\gamma(t s)=\delta_{t} \gamma(s)$ for $s, t>0$, or equivalently,

$\gamma(t)=\delta_{t} e, t>0$, where $e$ is a fixed vector in $R^{n}$.

It follows from (1-1) to (1-3) that when $t>0$,

$(1-5) \delta_{t}(x)=\exp \{A \ln t\} x$

for some real matrix $A$. Moreover since $\delta_{t}(x) \rightarrow 0$ as $t \rightarrow 0$, each eigenvalue of $A$ has positive real part. We shall set $a=\operatorname{tr} A$.

We remark that $\gamma(t)$ is homogeneous with respect to $\delta_{t}$ if and only if

$$
\gamma^{\prime}(t)=A \gamma(t) / t
$$

It is then immediate from (1-5) that $\delta_{t}$ has the following property:

(1-6) For each $x$ with $|x|=1$

$$
c_{1} t^{\alpha_{1}}<\left|\delta_{t} x\right|<c_{2} t^{\alpha_{2}} \quad \text { for } t \geqslant 1 \text {, }
$$

and

$$
c_{3} t^{\alpha_{3}}<\left|\delta_{t} x\right|<c_{4} t^{\alpha_{4}} \text { for } t<1
$$

here $c_{1}, c_{2}, c_{3}, c_{4}, \alpha_{1}, \alpha_{2}, \alpha_{3}$, and $\alpha_{4}$ are some positive constants. (To see the 
validity of the right-hand side of (1-6) extend $A$ to act on $C^{n}$ in the obvious way,

$$
A\left(x_{1}+i x_{2}\right)=A x_{1}+i A x_{2} \text {. }
$$

Then put $A$ in Jordan canonical form. The left-hand side of (1-6) follows from the right-hand side and the observation

$$
|x|=\left|\delta_{t}-1 \delta_{t} x\right| \leqslant\left|\delta_{t-1}\right|\left|\delta_{t} x\right| \text {.) }
$$

We wish next to define a norm function $\rho(x)$, which is smooth and positive away from the origin and which is homogeneous with respect to the dilations $\delta_{t}$; that is such that

$$
\rho\left(\delta_{t} x\right)=t \rho(x) \text { for } t>0 .
$$

If $\left|\delta_{t} x\right|$ were strictly monotonic, then $\left|\delta_{t} x\right|=1$ for a unique value of $t$. We might then define $\rho(x)$ to be the reciprocal of this unique $t$. Although $\left|\delta_{t} x\right|$ need not be monotonic, we have the following:

Proposition 1-7. There is a positive definite symmetric matrix $B$ such that

$$
\left\langle\delta_{t} x\right\rangle=\left\langle\delta_{t} x\right\rangle_{B}=\left(B \delta_{t} x, \delta_{t} x\right)^{1 / 2}
$$

is strictly increasing.

Proof. (The following proof was suggested to us by Charles McCarthy.)

$$
\frac{d}{d t}\left(B \delta_{t} x, \delta_{t} x\right)=\frac{1}{t}\left(\left(B A+A^{*} B\right) \delta_{t} x, \delta_{t} x\right) .
$$

Hence it suffices to find a real positive definite symmetric matrix $B$, such that $B A+A^{*} B=I$. Clearly

does the job.

$$
B=\int_{0}^{\infty} \exp \left(-t A^{*}\right) \exp (-t A) d t
$$

In view of Proposition 1-7, we may make the following definition:

Definition 1-8. For $x \neq 0, \rho(x)$ is the unique positive $t$ such that $\left\langle\delta_{t-1}(x)\right\rangle$ $=\left\langle\delta_{t-1}(x)\right\rangle_{B}=1$. For $x=0$ set $\rho(x)=0$. Clearly for $x \neq 0$

$$
x=\delta_{\rho(x)} \omega(x)
$$

where $\langle\omega(x)\rangle=1 ; \omega(x)$ is unique.

REMARK. We shall denote by $\rho^{*}(\xi)$ the distance function corresponding to the group $\delta_{t}^{*}=\exp \left(\ln t A^{*}\right)$. Then $\xi=\delta_{\rho^{*}(\xi)}^{*}\left(\omega^{*}(\xi)\right)$ where

$$
\left\langle\omega^{*}(\xi)\right\rangle^{2}=\left(B_{1} \omega^{*}(\xi), \omega^{*}(\xi)\right)=1
$$

for an appropriate positive definite symmetric matrix $B_{1}$.

The following proposition establishes the main properties of $\rho$ (and hence similarly of $\rho^{*}$ ).

Proposition 1-9.

(i) $\rho\left(\delta_{t} x\right)=t \rho(x), t>0$.

(ii) $\rho(x+y)<c(\rho(x)+\rho(y))$ for some $c>0$.

(iii) $\rho(x)$ is continuous and is $C^{\infty}$ in $R^{n}-0$.

(iv) $c_{1}|x|^{\alpha_{1}}<\rho(x)<c_{2}|x|^{\alpha_{2}}$ if $\rho(x) \geqslant 1$, and $c_{3}|x|^{\alpha_{3}}<\rho(x)<c_{4}|x|^{\alpha_{4}}$ if $\rho(x)<1$; 
here $c_{1}, c_{2}, c_{3}, c_{4}, \alpha_{1}, \alpha_{2}, \alpha_{3}$, and $\alpha_{4}$ are positive constants.

(v) Let us coordinatize $R^{n}$ by $\rho$ and $\omega$ where $\rho=\rho(x)$ and $\omega=\delta_{\rho}^{-1} x$. Then the volume element in $R^{n}$ is

$$
d x=\rho^{a-1} d \omega d \rho
$$

where d $\omega$ is a $C^{\infty}$ measure on the ellipsoid $(B \omega, \omega)=1, a=\operatorname{Tr} A$.

(vi) There is an $\eta>0$ such that for any $j>0$,

$$
\left|D^{j} \rho(x)\right| \leqslant c_{j}|\rho(x)|^{1-\eta j}
$$

where $D^{j}$ is a differentiation of order $j$.

Proof. (i) is obvious. To prove (ii) let $u=\rho(x)$ and $v=\rho(y)$, so $x=$ $\delta_{u} \omega(x)$ and $y=\delta_{v} \omega(y)$. Then for $\varepsilon>0$ and sufficiently small

$$
\begin{aligned}
& \left\langle\delta_{\varepsilon /(u+v)}(x+y)\right\rangle=\left\{\left\langle\delta_{\varepsilon /(u+v)} x\right\rangle+\left\langle\delta_{\varepsilon /(u+v)} y\right\rangle\right\} \\
& =\left\langle\delta_{e u /(u+v)} \omega(x)\right\rangle+\left\langle\delta_{\varepsilon v /(u+v)} \omega(y)\right\rangle \\
& \leqslant c\left\{\left(\frac{\varepsilon u}{u+v}\right)^{\alpha}+\left(\frac{\varepsilon v}{u+v}\right)^{\alpha}\right\} \leqslant c \varepsilon^{\alpha}
\end{aligned}
$$

for some positive $\alpha$. Thus if $\varepsilon$ is sufficiently small

$$
\left\langle\delta_{\varepsilon /(u+v)}(x+y)\right\rangle<1 .
$$

Thus

$$
\rho(x+y) \leqslant \frac{u+v}{\varepsilon}=\frac{1}{\varepsilon}(\rho(x)+\rho(y)) .
$$

It is clear that $\rho$ is continuous. To see that $\rho(x)$ is $C^{\infty}$ away from the origin apply the implicit function theorem to

$$
\begin{aligned}
F(x, t) & =\left\langle\delta\left(t^{-1}\right) x\right\rangle^{2} \\
\left(\frac{\partial F}{\partial t}\right. & \left.=-\frac{1}{t}\left|\delta\left(t^{-1} x\right)\right|^{2}\right) .
\end{aligned}
$$

The inequalities (iv) follow from (1-4).

To see (v), note

$$
x=\exp (A \ln \rho) \omega .
$$

Let us suppose we are in some region where one of the coordinates of $\omega$ (say $\omega_{n}$ ) can be expressed in terms of the other $n-1$. Then the rows of the Jacobian matrix are expressed as follows:

$$
\frac{\partial x}{\partial \rho}=\left(\frac{\partial x_{1}}{\partial \rho}, \ldots, \frac{\partial x_{n}}{\partial \rho}\right)=\frac{A}{\rho} \exp (A \ln \rho) \omega,
$$

and for $1<j<n-1$

$$
\frac{\partial x}{\partial \omega_{j}}=\left(\frac{\partial x_{1}}{\partial \omega_{j}}, \ldots, \frac{\partial x_{n}}{\partial \omega_{j}}\right)=\exp (A \ln \rho) \omega^{(j)}
$$

where $\omega^{(j)}$ is a smooth vector function of $\omega_{1}, \ldots, \omega_{n-1}$. The result (v) then follows by taking determinants. (vi) is an easy consequence of the homogeneity and smoothness of $\rho$. 
We now describe a group of dilations and a norm function associated to a sufficiently large class of smooth curves $\gamma(t)$ with $\gamma(0)=0$.

Definition 1-10. We shall say that a curve $\gamma(t)$ is of standard type if

$$
\gamma(t)=\left(\gamma_{1}(t), \ldots, \gamma_{n}(t)\right)
$$

where

$$
\gamma_{k}(t)=\frac{1}{j_{k} !} t^{j_{k}}+\text { higher terms, with } 1 \leqslant j_{1}<j_{2}<\cdots<j_{n} .
$$

Let $\gamma(t)$ be of standard type. Then by the dilation group, $\delta_{t}$, and norm function, $\rho$, corresponding to $\gamma$, we mean those corresponding to the matrix $A=\operatorname{diag}\left(j_{1}, \ldots, j_{n}\right), \gamma$ will be approximately homogeneous: Namely $\delta_{h}^{-1} \gamma(h t) \sim \gamma(t)$ for small $h$ and $t$. This means that arguments for homogeneous curves will apply to curves of standard type (at least for small $t$ and h). Moreover for many purposes it will suffice to consider curves of standard type. This fact is contained in the following proposition:

Proposition 1-12. Let $\bar{\gamma}(t)$ be a $C^{\infty}$ curve in $R^{n}$ with $\bar{\gamma}(0)=0$ such that

$$
\left.\frac{d^{j} \bar{\gamma}(t)}{d t^{j}}\right|_{t=0}, \quad j=1,2, \ldots,
$$

span $R^{n}$. Then

$$
\bar{\gamma}(t)=c \gamma(t)
$$

where $C$ is a constant nonsingular $n \times n$ matrix, and $\gamma(t)$ is a curve of standard type.

Proof. It suffices to find $n$ vectors, $e_{1}, \ldots, e_{n}$, spanning $R^{n}$ such that

$$
\bar{\gamma}(t)=\gamma_{1}(t) e_{1}+\cdots+\gamma_{n}(t) e_{n}
$$

where the $\gamma_{i}(t)$ satisfy $(1-11)$. To this end, set

$$
j_{1}=\inf \left\{j \mid \bar{\gamma}^{(j)}(0) \neq 0\right\} \text {, }
$$

and given $j_{1}, \ldots, j_{k}, k<n$, define

$$
j_{k+1}=\inf \left\{j \mid j>j_{k} \text { and }\left\{\bar{\gamma}^{\left(j_{1}\right)}(0), \ldots, \bar{\gamma}^{\left(j_{k}\right)}(0), \bar{\gamma}^{j}(0)\right\}\right.
$$

are linearly independent $\}$.

We then take $e_{k}=\bar{\gamma}^{\left(j_{k}\right)}(0)$.

2. Estimates for trigonometric integrals. $\$ 2$ contains the estimates for trigonometric integrals which are basic for most of this paper.

THEOREM 1. Let $\gamma(t), t>0$, be a curve in $R^{n}$ which satisfies $\gamma^{\prime}(t)=A \gamma(t) / t$ for some fixed matrix $A$. Assume $\{\gamma(t)\}$ does not lie in an affine hyperplane. Then for $0<b<d$

$$
\left|\int_{b}^{d} \exp \{i \xi \cdot \gamma(t)\} d t\right| \leqslant c\|\xi\|^{-1 / n}
$$


where $c$ remains bounded as long as $b$ and $d$ lie in a compact subinterval of $(0, \infty)$.

Assume now that the eigenvalues of $A$ have positive real part. Let $\delta_{t}$ and $\delta_{t}^{*}$ be the dilations associated with $A$ and $A^{*}$, and let $\rho^{*}$ be the corresponding norm function (to $\delta_{t}^{*}$ ) as described in $\$ 1$. Then we have the following simple consequence of Theorem 1 .

COROLlaRY. Under the assumptions of Theorem 1

$$
\left|\int_{b}^{d} \exp \{i \xi \cdot \gamma(t h)\} d t\right| \leqslant c\left(\rho^{*} \delta_{h}^{*}(\xi)\right)^{-\sigma}
$$

for some positive $\sigma$ all $\xi \neq 0$, and $h>0$.

(The corollary follows from Theorem 1 by observing that if $\gamma^{\prime}(t)=$ $A \gamma(t) / t$, then $\gamma(t h)=e^{A \log h} \gamma(t)=\delta_{h}(\gamma(t))$, and $\rho^{*}(\xi)<c_{2}|\xi|^{\alpha_{2}}$ if $|\xi|>1$. (See Proposition 1-9 (iv).) So 2.1 holds with $\sigma=1 / \alpha_{2} n$.)

The corollary gives the necessary estimate for "homogeneous" curves. We shall need a corresponding estimate for smooth curves. If $\gamma$ is a curve of standard type, we let $\rho$ and $\delta_{h}$ be the associated norm function and dilation group. (See \$1.)

THEOREM 2. Let $\gamma(t)$ be a curve of standard type. Then

$$
\left|\int_{b}^{d} \exp \{i \xi \cdot \gamma(t h)\} d t\right| \leqslant \frac{c}{\left(\rho\left(\delta_{h}(\xi)\right)\right)^{\sigma}}
$$

for some positive $c$ and $\sigma$, provided $0 \leqslant b \leqslant d \leqslant d_{0}$ and $h<h_{0}$, where $d_{0}$ and $h_{0}$ are sufficiently small.

The proofs of Theorems 1 and 2 depend upon the following lemma due to van der Corput.

LeMma 2-3 (VAN DER CoRPuT). Let

$$
\left|d^{j} f(t) / d t^{j}\right| \geqslant \lambda>0
$$

for some positive integral value of $j$ and $b<t<d$. If $j=1$ assume in addition that $f^{\prime}(t)$ is monotonic. Then

$$
\left|\int_{b}^{d} \exp \{i f(t)\} d t\right| \leqslant c(j) \lambda^{-1 / j}
$$

Lemma 2-3 is proved in Zygmund [1959, vol. I, p. 197] for the cases $j=1$ and 2 . The lemma for general $j$ follows by a similar inductive argument.

Proof of Theorem 1. Let $\psi(t)=\gamma\left(e^{t}\right)$ so that $\psi(t)$ satisfies the differential equation $\psi^{\prime}(t)=A \psi(t)$. By changing variables and integrating by parts, we see that it suffices to prove

$$
\left|\int_{\alpha}^{\beta} \exp \{i \lambda \xi \cdot \psi(t)\} d t\right| \leqslant \frac{c}{\lambda^{1 / n}},
$$

for $\xi$ on the unit sphere and $\lambda>0$.

We shall first prove (2-4) under the assumption that none of the matrices $A$, 
$A^{2}, \ldots, A^{n}$ have 1 as an eigenvalue.

Set $u(t)=\xi \cdot \psi(t)$. We note that $u^{\prime}(t)=\xi \cdot \psi^{\prime}(t)$ satisfies an $n$th order differential equation; for if $\sum_{j=0}^{n} c_{j} x^{j}$ is the characteristic polynomial of $A$,

$$
\sum_{j=0}^{n} c_{j} \frac{d^{j}}{d t^{j}} u^{\prime}(t)=\sum_{j=0}^{n} c_{j}\left(\xi \cdot A^{j} \psi^{\prime}(t)\right)=0 .
$$

Thus if $u^{\prime}\left(t_{0}\right)=\cdots=u^{(n)}\left(t_{0}\right)=0, u^{\prime}(t) \equiv 0$. Hence if $\xi \cdot \psi^{(j)}\left(t_{0}\right)=0, j=1$, $2, \ldots, n, u(t)$ is constant, contradicting the assumption that $\gamma(t)$ not lie in any affine hyperplane.

Now invoking a compactness argument, we have, under the assumptions of Theorem 1,

$$
\sum_{j=1}^{n}\left|\frac{d^{j}}{d t^{j}}(\lambda \xi \cdot \psi(t))\right| \geqslant c \lambda, \quad c>0
$$

for $|\xi|=1, \lambda>0$, and $\alpha \leqslant t \leqslant \beta$. So Lemma 2.3 will imply 2.4 if we can divide the interval $(\alpha, \beta)$ into a uniformly bounded (in $\lambda$ and $\xi$ ) number of subintervals in each of which $d(\lambda \xi \cdot \psi(t)) / d t$ is monotone and a particular fixed one of the first $n$ derivatives of $\lambda \xi \cdot \psi(t)$ is larger than or equal to the others. Thus it suffices to prove (2-5) and (2-6) below.

$(2-5) u^{\prime \prime}(t)$ has only a bounded number of zeros on $(\alpha, \beta)$ (i.e. a bounded number independent of $\xi$ for $\xi$ in a compact set), and

(2-6) The number of zeros of $u^{(j)}(t)-u^{(k)}(t)$ is bounded for $\alpha<t<\beta$, $1<j<k<n$, and $\xi$ on the unit sphere.

Let $\psi(t)=\left(\psi_{1}(t), \ldots, \psi_{n}(t)\right)$. Each $\psi_{j}(t)$ is the restriction to real $t$ of a holomorphic function. If (2-5) were false there would be a sequence $\xi_{1}$, $\xi_{2}, \ldots$ with $\left|\xi_{r}\right|=1$ such that the number of zeros of $\xi_{r} \cdot \psi^{\prime \prime}(t)$ is greater than $r$. By passing to a subsequence we may assume $\xi_{r} \rightarrow \xi^{\prime}$ for some $\xi^{\prime}$ with $\left|\xi^{\prime}\right|=1$. Then by the argument principle we would obtain

$$
\xi^{\prime} \cdot \psi^{\prime \prime}(t) \equiv 0
$$

That is $0 \equiv\left(\xi^{\prime} \cdot A^{2} \psi(t)\right) \equiv\left(A^{* 2} \xi^{\prime} \cdot \psi(t)\right) \equiv 0$. This will be in contradiction to the hypothesis if we can show that $\boldsymbol{A}$ is nonsingular. But if $\boldsymbol{A}$ were singular, $A^{*} \xi^{\prime}$ would be zero for some nonzero $\xi^{\prime}$. Then $0 \equiv \xi^{\prime} \cdot A \psi(t) \equiv \xi^{\prime} \cdot \psi^{\prime}(t)$. Thus $\xi^{\prime} \cdot \psi(t)$ would be constant contrary to the hypothesis.

PROOF OF (2-6). If the number of zeros of $u^{(j)}(t)-u^{(k)}(t)$ were not bounded, the above argument would give a $\xi^{\prime} \neq 0$ such that

$$
u^{(j)}(t)-u^{(k)}(t) \equiv 0 \text {. }
$$

Thus

$$
\left(\xi^{\prime} \cdot A^{j} \psi(t)\right) \equiv\left(\xi^{\prime} \cdot A^{k} \psi(t)\right) .
$$

Hence

$$
\left(A^{j^{*}} \xi^{\prime}-A^{k^{*}} \xi^{\prime}\right) \cdot \psi(t) \equiv 0 .
$$

Thus

$$
A^{j^{*}} \xi^{\prime}-A^{k^{*}} \xi^{\prime} \equiv 0
$$

(from the hypothesis of the theorem). 
Thus

$$
A^{(k-j)^{*}} A^{j^{*}} \xi^{\prime}=A^{j^{*}} \xi^{\prime}
$$

Since $A$ is nonsingular $A^{j^{*}} \xi^{\prime}$ is an eigenvector of $A^{(k-j)^{*}}$ with eigenvalue 1 . However we are assuming $A^{k-j}$ does not have 1 as an eigenvalue, giving us a contradiction.

To obtain (2-4) without the hypothesis on the eigenvalue of $A, A^{2}, \ldots, A^{n}$, we make change of variables $t=s / \nu$ in (2-4), where $\nu>0$ is such that 1 is not an eigenvalue of $A / \nu, A^{2} / \nu^{2}, \ldots, A^{n} / \nu^{n}$. Then set $\bar{\psi}(t)=\psi(t / \nu)$. We have $\bar{\psi}^{\prime}(t)=A \bar{\psi}(t) / \underline{v}$. We can now apply the above argument to obtain (2-4) with $\psi$ replaced by $\bar{\psi}, \alpha$ replaced by $\alpha \nu$ and $\beta$ replacec by $\beta \nu$. Then (2-4) will follow by a change of variables.

Proof OF TheORem 2. Assume first $\gamma^{\prime}(0)=0$, so that each $j_{k}>1$. Let

$$
M=\max _{k=1,2, \ldots, n}\left|\xi_{k} \cdot h^{j_{k}}\right| \text {, }
$$

and let $k_{0}$ be an integer such that

$$
M=\left|\xi_{k_{0}} h^{j_{k_{0}}}\right|
$$

Set

$$
f(t)=\xi \cdot \gamma(t h) \text {. }
$$

By Lemma (2-3), it suffices to show that for $t<d_{0}$ and $h<h_{0}$, the following statement is true:

$$
\left|d^{j_{k_{0}}} f(t) / d t^{j_{k_{0}}}\right| \geqslant M / 2
$$

ProOf OF (2-7).

$$
\begin{aligned}
f^{\left(j_{k_{0}}\right)}(t)= & h^{j_{k_{0}}} \sum_{1<l<k_{0}} \xi_{l} \gamma_{l}^{\left(j_{k_{0}}\right)}(t h) \\
& +h^{j k_{0} \xi_{k_{0}}} \gamma_{k_{0}}^{\left(j_{k_{0}}\right)}(t h)+h^{j_{k_{0}}} \sum_{l>k_{0}} \xi_{l} \gamma l^{\left(j_{k_{0}}\right)}(t h) .
\end{aligned}
$$

We shall show that the first and third terms above are less than $M / 8$, while the second term is greater than $3 M / 4$ for $t$ and $h$ sufficiently small. For $1<l<k_{0}$,

$h^{j_{k_{0}}}\left|\xi_{l} \gamma \gamma^{\left(j_{k_{\theta}}\right)}(t h)\right|<c h^{j_{k_{0}}}\left|\xi_{l}\right|=c h^{j_{l}}\left|\xi_{l}\right| h^{j_{k}-j_{l}}<c h M<M / 8$ if $h$ is sufficiently small.

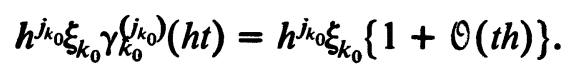

Thus

$$
\left|h^{j_{k_{0}}} \xi_{k_{0}} \gamma_{k_{0}}^{j_{k_{0}}}(h t)\right|>3 M / 4
$$

if $t$ and $h$ are sufficiently small. Finally, for $l>k_{0}$

$$
\begin{aligned}
\left|h^{j_{k_{0}} \xi l} \gamma l^{\left(j_{k_{0}}\right)}(t h)\right| & <c h^{j_{k_{0}}\left|\xi_{l}\right| t^{j_{l}-j_{k_{0}}} h^{j_{l}-j_{k_{0}}}} \\
& <c M t^{j_{l}-j_{k_{0}}} \quad(\text { if } h<1) \\
& <M / 8,
\end{aligned}
$$

if $t$ is sufficiently small. This completes the proof of (2-7). 
If $\gamma^{\prime}(0) \neq 0$, we first make a change of variables $t=u^{2}$, and then we apply the above argument.

3. The a priori $L^{2}$ estimates.

(a) The Hilbert transform. Recall that we have called a curve $\gamma(t)$ defined for positive $t$ homogeneous if it satisfies a differential equation

$$
\gamma^{\prime}(t)=A \gamma(t) / t, \quad t>0,
$$

where $A$ is a real $n \times n$ matrix such that each eigenvalue of $A$ has positive real part. Equivalently,

$$
\gamma(t)=\delta_{t} e=\exp \{A \ln t\} e, \quad t>0,
$$

for some vector $e$. In defining the Hilbert transform corresponding to a curve $\gamma(t)$, we must prescribe $\gamma(t)$ for all real values of $t$. Of course we can extend $\gamma(t)$ to negative values of $t$ by setting

$$
\gamma(t)=\delta_{(-t)} f, \quad t<0,
$$

for some vector $f$. The question is now whether we are required to have some further relation between $\gamma(t)$ for $t>0$ and $\gamma(t)$ for $t<0$. We shall see that we need to assume

$$
\{\xi \mid \xi \cdot \gamma(t) \equiv 0, t>0\}=\{\xi \mid \xi \cdot \gamma(t) \equiv 0, t<0\} .
$$

We are thus led to the following definition:

Definition 3-1. A curve $\gamma(t)$ defined for all real $t$ will be called $t w o$-sided homogeneous if the following two conditions hold.

$$
\gamma(t)= \begin{cases}\delta_{t} e, & t>0 \\ \delta_{-t} f, & t<0 \\ 0, & t=0\end{cases}
$$

(Here $\delta_{t}$ is a one parameter group of dilations and $e$ and $f$ are vectors in $R^{n}$.)

$$
\{\xi \mid \xi \cdot \gamma(t) \equiv 0, t>0\}=\{\xi \mid \xi \cdot \gamma(t) \equiv 0, t<0\},
$$

i.e. the linear space spanned by $\{\gamma(t)\}_{t>0}=$ linear space spanned by $\{\gamma(t)\}_{t<0}$.

If $\gamma(t)$ is a continuous curve, defined for $\varepsilon \leqslant|t| \leqslant N$, we set

$$
\mathcal{K}_{\varepsilon, N} f(x)=\int_{\varepsilon<|t|<N} f(x-\gamma(t)) \frac{d t}{t}, \text { for } f \text { in } \mathcal{S} .
$$

We shall now prove

THEOREM 3. (A) Let $\gamma(t)$ be a two-sided homogeneous curve. Then

$$
\left\|\mathcal{H}_{\varepsilon, N} f\right\|_{2} \leqslant c(\gamma)\|f\|_{2} \text {. }
$$

Also as $\varepsilon \rightarrow 0$ and $N \rightarrow \infty, \mathcal{H}_{\varepsilon, N} f$ converges in $L^{2}$ to an $L^{2}$ function $\mathcal{H} f$. Furthermore,

$$
\|\mathcal{H} f\|_{2} \leqslant c(\gamma)\|f\|_{2} \text {. }
$$

(B) Assume $\gamma(t)$ is a $C^{\infty}$ curve such that for small $t$ it lies in the subspace spanned by $\left\{\gamma^{(j)}(0)\right\}_{j=1}^{\infty}$. Then the conclusions of part (A) hold also in this case for $\mathcal{H}_{\varepsilon, 1}$, when $\varepsilon \rightarrow 0$. 
REMARK 3-5. The conclusion of Theorem 3 part (A) is false if $\gamma(t)$ satisfies (3-2) but not (3-3).

Proof of (A). We first prove part (A) under the additional hypothesis that $\xi \cdot \gamma(t) \equiv 0$ implies $\xi=0$ (i.e. $\gamma$ does not lie in a proper subspace).

A simple calculation shows that

$$
\widehat{\mathcal{F}_{e, N} f}(\xi)=m_{e, N}(\xi) \hat{f}(\xi)
$$

where

$$
m_{\varepsilon, N}(\xi)=\int_{\varepsilon<|t|<N} \exp (-2 \pi i \gamma(t) \cdot \xi) \frac{d t}{t} .
$$

Conclusion (i) is equivalent to the uniform boundedness of $m_{\varepsilon, N}(\xi)$. A homogeneity argument (i.e. replacing $\xi$ by $\delta_{\rho^{*}}^{*}(\xi) \omega^{*}(\xi)$ and changing variables $\left.s=t \rho^{*}(\xi)\right)$ allows us to assume $\rho^{*}(\xi)=1$. Then

$$
\begin{aligned}
& \left|\int_{\varepsilon<|t|<1} \exp (-2 \pi i \gamma(t) \cdot \xi) \frac{d t}{t}\right| \\
& \quad<\int_{\varepsilon<|t|<1}|\exp (-2 \pi i \gamma(t) \cdot \xi)-1| \frac{d t}{|t|} \leqslant c(\gamma)
\end{aligned}
$$

since $|\gamma(t)|<c|t|^{\alpha}$ for some positive $\alpha$, near $t=0$ (see (1-6)).

Now for $\nu>0$

$$
\begin{aligned}
\mid \int_{2^{\nu}<t<2^{p+1}} & \exp (-2 \pi i \gamma(t) \cdot \xi) \frac{d t}{t} \mid \\
& =\left|\int_{1<t<2} \exp \left(-2 \pi i \gamma\left(2^{\nu} t\right) \cdot \xi\right) \frac{d t}{t}\right| \\
& =\left|\int_{1<t<2} \exp \left(-2 \pi i \gamma(t) \cdot \delta_{2^{*}}^{*} \xi\right) \frac{d t}{t}\right| \leqslant \frac{c(\gamma)}{2^{\sigma \nu}}
\end{aligned}
$$

for some $\sigma>0$ by 2.1 .

Similarly, if $1<\alpha<\beta<2 \alpha$

$$
\left|\int_{\alpha<|t|<\beta} \exp (-2 \pi i \gamma(t) \cdot \xi) \frac{d t}{t}\right| \leqslant \frac{c(\gamma)}{\alpha^{\sigma}} .
$$

Thus adding the terms shows

$$
\left|m_{\varepsilon, N}(\xi)\right| \leqslant c(\gamma)
$$

Thus (i) follows. The remaining parts of Theorem 3 (A) are now easy. We shall now remove the restriction that $\gamma$ does not lie in a proper subspace. Let $V_{1}=\{\xi \mid \xi \cdot \gamma(t) \equiv 0\}$, and set $V_{2}=V_{1}^{\perp}$. Then $\gamma(t)$ is in $V_{2}$, and for $\xi$ in $V_{2}$, $\xi \cdot \gamma(t)=0$ implies $\xi=0$. Note that $V_{1}$ is invariant under $A^{*}$. For $\xi \cdot \gamma(t) \equiv 0$ implies

$$
\xi \cdot \gamma^{\prime}(t) \equiv 0 \Rightarrow \xi \cdot A \gamma(t) \equiv 0 \Rightarrow A^{*} \xi \cdot \gamma(t) \equiv 0 .
$$

Thus $V_{2}$ is invariant under $A$. Let $A_{1}$ be the restriction of $A$ to $V_{2}$. Then

$$
\gamma^{\prime}(t)=A_{1} \gamma(t) / t,
$$


and each eigenvalue of $A_{1}$ has positive real part. For $x$ in $R^{n}$, let $x=x_{1}+x_{2}$ with $x_{i}$ in $V_{i}$. Fix $x_{1}$ and let

$$
\mathcal{F}_{\varepsilon, N}^{x_{1}} f\left(x_{2}\right)=\int_{\varepsilon<|t|<N} f\left(x_{1}+\left(x_{2}-\gamma(t)\right)\right) \frac{d t}{t} .
$$

Then we apply our previous argument in the $x_{2}$ variable first, and then integrate in $x_{1}$ to obtain the result.

The proof of part (B) in Theorem 3 is similar. If the vectors $\gamma^{(j)}(0)$ span $R^{n}$, we may assume $\gamma$ is of standard type by Proposition 1-12. The homogeneity argument which allowed us to assume $\rho^{*}(\xi)=1$ has to be modified as follows: We divide the integral for $m_{\varepsilon, 1}(\xi)$ into $t\left\langle(\rho(\xi))^{-1}\right.$ and $t>1 / \rho(\xi)$. We then argue as above using (2-2) instead of (2-1).

Thus for $t_{0}$ sufficiently small

$$
\begin{aligned}
& \left|\int_{\substack{|t|<1 / \rho(\xi) \\
|t|<t_{0}}} \exp (2 \pi i \xi \cdot \gamma(t)) \frac{d t}{t}\right| \\
& \quad<\int_{\substack{|t|<1 / \rho(\xi) \\
|t|<t_{0}}}|\exp (2 \pi i \xi \cdot \gamma(t))-1| \frac{d t}{|t|}<c \sum_{j=1}^{n}\left|\xi_{j}\right| \int_{0}^{1 / \rho(\xi)} t^{k_{j}-1} d t \\
& \quad<c \sum_{j=1}^{n}\left|\xi_{j}\right|(\rho(\xi))^{-k_{j}}<c .
\end{aligned}
$$

Now let $\eta$ be a small number depending on $\gamma$, and let $t_{0}$ be a small number depending on $\gamma$ and $\eta$. Then if $1<\alpha<\beta<2 \alpha$,

$$
\begin{aligned}
\left|\int_{\substack{\alpha / \rho(\xi)<|t|<\beta / \rho(\xi) \\
|t|<t_{0}}} \exp \{2 \pi i \xi \cdot \gamma(t)\} \frac{d t}{t}\right| \\
\quad=\left|\int_{\substack{1<|t|<\beta / \alpha \\
|t|<t_{0} \rho(\xi) / \alpha}} \exp \left\{2 \pi i \xi \cdot \gamma\left(t \frac{\alpha}{\rho(\xi)}\right)\right\} \frac{d t}{t}\right| \\
=\left|\int_{\substack{\eta<|t|<\eta \beta / \alpha \\
|t|<\eta t_{0} \rho(\xi) / \alpha}} \exp \left\{2 \pi i \xi \cdot \gamma\left(\frac{1}{\eta} t \frac{\alpha}{\rho(\xi)}\right)\right\} \frac{d t}{t}\right| \leqslant c_{\eta} \frac{1}{\alpha^{\sigma}},
\end{aligned}
$$

if $\eta$ is sufficiently small and $t_{0}$ is sufficiently small (since $\alpha / \rho(\xi)<t_{0}$ ). Having fixed $t_{0}$,

$$
\left|\int_{t_{0}<|t|<1} \exp (2 \pi i \xi \cdot \gamma(t)) \frac{d t}{t}\right| \leqslant c .
$$

The proof when $\gamma^{(j)}(0)$ do not span $R^{n}$ then is completed as in part (A).

Proof OF Remark 3-5. Let 


$$
\begin{aligned}
& U=\{\xi \mid \xi \cdot \gamma(t)=0 \text { for } t>0\} \quad \text { and } \\
& V=\{\xi \mid \xi \cdot \gamma(t)=0 \text { for } t<0\} .
\end{aligned}
$$

Assume there is a $\xi$ in $U$, so that $\xi$ is not in $V$, and take $|\xi|=1$. Then $\xi=\xi_{1}+\xi_{2}$ with $\xi_{1}$ in $V$ and $\xi_{2}$ in $V^{\perp}$. The arguments proving Theorem 3 part (A) then show

$$
\left|\int_{|t|<1} \exp [2 \pi i \gamma(t) \cdot \xi] \frac{d t}{t}\right| \leqslant c
$$

and

$$
\begin{aligned}
\left|\int_{-N<t<-1} \exp [2 \pi i \gamma(t) \cdot \xi] \frac{d t}{t}\right| \\
\quad=\left|\int_{-N<t<-1} \exp \left[2 \pi i \gamma(t) \cdot \xi_{2}\right] \frac{d t}{t}\right| \leqslant c .
\end{aligned}
$$

On the other hand clearly

$$
\int_{1}^{N} \exp (2 \pi i \gamma(t) \cdot \xi) \frac{d t}{t}=\log _{e} N
$$

and so $m_{1, N}(\xi)$ is unbounded as $N \rightarrow \infty$.

(b) The Maximal function. Let $\gamma(t)$ be a continuous curve defined for $t \geqslant 0$. For $f$ in $\mathcal{S}$ we set

$$
\begin{gathered}
\Re(x)=\sup _{h>0} \frac{1}{h}\left|\int_{0}^{h} f(x-\gamma(t)) d t\right|, \text { and } \\
\Re_{1} f(x)=\sup _{1>h>0} \frac{1}{h}\left|\int_{0}^{h} f(x-\gamma(t)) d t\right| .
\end{gathered}
$$

Here we prove,

THEOREM 4. (A) Let $\gamma(t)$ be homogeneous; then

$$
\|\Re f\|_{2} \leqslant c(\gamma)\|f\|_{2} \text {. }
$$

(B) Let $\gamma(t)$ be $C^{\infty}$. Assume an initial segment of $\gamma(t)$ lies in the subspace spanned by $\left\{\gamma^{j}(0)\right\}_{j=1}^{\infty}$. Then

$$
\left\|\Re_{1} f\right\|_{2} \leqslant c(\gamma)\|f\|_{2}
$$

The crux of the proof of Theorem 4 are estimates for appropriate $g$ functions which we state as a separate theorem. First some notation. We set

$$
N_{h} f(x)=\frac{1}{h} \int_{h}^{2 h} f(x-\gamma(t)) d t
$$

We let $\phi$ be a $C_{0}^{\infty}$ function with $\hat{\phi}(0)=1$, and

$$
\phi_{h}(x)=\left(\operatorname{det} \delta_{h}\right)^{-1} \phi\left(\delta_{h}^{-1} x\right),
$$

for the appropriate dilation group $\delta_{h}$. If $\gamma(t)$ is homogeneous and $f$ is in $\delta$, we define $g(f)(x)$ by 


$$
g^{2} f(x)=\int_{0}^{\infty} \frac{d h}{h}\left|N_{h} f(x)-\phi_{h} * f(x)\right|^{2},
$$

where the underlying group of dilations is the obvious group associated to $\gamma(t)$. If $\gamma(t)$ is of standard type and $f$ is in $\delta$, we define

$$
g_{1}^{2} f(x)=\int_{0}^{1} \frac{d h}{h}\left|N_{h} f(x)-\phi_{h} * f(x)\right|^{2} .
$$

Here the underlying group of dilations is that associated with a curve of standard type. (See §1.)

THEOREM 5. (A) If $\gamma$ is homogeneous and $\gamma$ does not lie in a proper subspace of $R^{n}$,

$$
\|g(f)\|_{2} \leqslant c(\gamma)\|f\|_{2} .
$$

(b) If $\gamma(t)$ is of standard type

$$
\left\|g_{1}(f)\right\|_{2} \leqslant c(\gamma)\|f\|_{2} .
$$

We shall first see how Theorem 5 implies Theorem 4. Here we shall restrict our discussion to part (A), the argument for part (B) being analogous. Using an argument in the proof of Theorem 3, we may suppose that $\gamma$ does not lie in a proper subspace of $R^{n}$. We may also assume $f \geqslant 0$. Now

$$
g^{2} f(x) \geqslant \sup _{\varepsilon>0} \frac{1}{\varepsilon} \int_{0}^{\varepsilon}\left|N_{h} f(x)-\phi_{h} * f(x)\right|^{2} d h .
$$

So by Schwarz's inequality

$$
g(f)(x) \geqslant \sup _{\varepsilon>0} \frac{1}{\varepsilon} \int_{0}^{\varepsilon}\left|N_{h} f(x)-\phi_{h} * f\right| d h .
$$

Thus

$$
\sup _{\varepsilon>0} \frac{1}{\varepsilon} \int_{0}^{\varepsilon}\left|N_{h} f(x)\right| d h \leqslant g(f)(x)+\sup _{h>0}\left|\phi_{h} * f(x)\right| .
$$

Also since $f \geqslant 0$,

$$
\begin{aligned}
\frac{1}{\varepsilon} \int_{0}^{\varepsilon} N_{h} f(x) d x & =\frac{1}{\varepsilon} \int_{0}^{\varepsilon} \frac{1}{h} \int_{h}^{2 h} f(x-\gamma(t)) d t d h \\
& \geqslant \frac{1}{\varepsilon} \int_{0}^{\varepsilon} f(x-\gamma(t)) \int_{t / 2}^{t} \frac{d h}{h} d t \\
& =\frac{\ln 2}{\varepsilon} \int_{0}^{\varepsilon} f(x-\gamma(t)) d t .
\end{aligned}
$$

Hence, taking the supremum in $\varepsilon$, we have

$$
\Re f(x) \leqslant c\left(g(f)(x)+\sup _{h>0} \phi_{h} * f(x)\right) .
$$

Now $\|g(f)(x)\|_{2} \leqslant c\|f\|_{2}$ by Theorem 5 , and

$$
\left\|\sup _{h>0} \phi_{h} * f\right\|_{2} \leqslant c\|f\|_{2}
$$

by standard arguments; see e.g. Theorem 2.3 of Rivière [1971]. (The Vitali 
family can be taken to be $U_{h}=\{x \mid \rho(x) \leqslant h\}$.) Thus

$$
\|\Re f\|_{2} \leqslant c\|f\|_{2} \text {. }
$$

We now turn to the proof of Theorem 5. Consider part (A).

$$
\begin{aligned}
\|g(f)\|_{2}^{2} & =\int_{0}^{\infty} \frac{d h}{h}\left\|N_{h} f(x)-\phi_{h} * f(x)\right\|_{2}^{2} \\
& =\int_{0}^{\infty} \frac{d h}{h}\left\|\widehat{N_{h} f}(\xi)-\widehat{\phi_{h} * f}(\xi)\right\|_{2}^{2} .
\end{aligned}
$$

Now $\widehat{\phi_{h} * f}(\xi)=\hat{\phi}\left(\delta_{h}^{*}(\xi)\right) \hat{f}(\xi)$, and a simple calculation shows

$$
\widehat{N_{h} f}(\xi)=n\left(\delta_{h}^{*} \xi\right) \hat{f}(\xi)
$$

where

$$
n(\xi)=\int_{1}^{2} \exp (-2 \pi i \xi \cdot \gamma(t)) d t
$$

Thus

$$
\|g(f)\|_{2}^{2}=\int|\hat{f}(\xi)|^{2} \int_{0}^{\infty}\left|n\left(\delta_{h}^{*} \xi\right)-\hat{\phi}\left(\delta_{h}^{*} \xi\right)\right|^{2} \frac{d h}{h} d \xi .
$$

Hence it remains to show

$$
G(\xi)=\int_{0}^{\infty} \frac{d h}{h}\left|n\left(\delta_{h}^{*} \xi\right)-\hat{\phi}\left(\delta_{h}^{*} \xi\right)\right|^{2}
$$

is uniformly bounded.

Writing $\xi=\delta_{\rho^{*}(\xi)}^{*} \omega^{*}(\xi)$, and changing variables, we see $G(\xi)=G\left(\omega^{*}(\xi)\right)$. Thus we may assume $\rho^{*}(\xi)=1$. As $\hat{\phi}(0)=1,\left|n\left(\delta_{h}^{*} \xi\right)-\hat{\phi}\left(\delta_{h}^{*} \xi\right)\right| \leqslant c h^{\sigma}$ for some positive $\sigma$. By (2-1)

$$
\left|n\left(\delta_{h}^{*} \xi\right)\right| \leqslant c / h^{\sigma}
$$

for some $\sigma>0$. Also

$$
\left|\hat{\phi}\left(\delta_{h}^{*} \xi\right)\right| \leqslant c / h^{\sigma} .
$$

Hence Theorem 5 part (A) follows easily.

Proof of part (B). Arguing as above, it suffices to show

$$
\int_{0}^{1}\left|m_{h}(\xi)-\hat{\phi}\left(\delta_{h}(\xi)\right)\right|^{2} \frac{d h}{h} \leqslant c,
$$

where

$$
\begin{aligned}
m_{h}(\xi) & =\frac{1}{h} \int_{h}^{2 h} \exp (-2 \pi i \xi \cdot \gamma(t)) d t \\
& =\int_{1}^{2} \exp (-2 \pi i \xi \cdot \gamma(t h)) d t .
\end{aligned}
$$

In the integral (3-6) only the part where $h$ is small is critical. If we divide that part into two further parts (where $h<1 / \rho(\xi)$, and $h \geqslant 1 / \rho(\xi)$ ), we see that (3-6) follows easily from four estimates 


$$
\begin{aligned}
\left|1-m_{h}(\xi)\right| & \leqslant c \sum_{k=1}^{n} h^{j_{k}}\left|\xi_{k}\right|, \\
\left|1-\hat{\phi}\left(\delta_{h}(\xi)\right)\right| & \leqslant c \sum_{k=1}^{n} h^{j_{k}}\left|\xi_{k}\right|, \\
\left|\hat{\phi}\left(\delta_{h}(\xi)\right)\right| & \leqslant c\left|\delta_{h}(\xi)\right|^{-\sigma}, \text { and } \\
\left|m_{h}(\xi)\right| & <c\left|\delta_{h}(\xi)\right|^{-\sigma}
\end{aligned}
$$

for small $h$ and for some $\sigma>0$. (3-7), (3-8), and (3-9) are easy; and (3-10) follows from (2-2).

(c) Maximal Hilbert transforms. Let $\gamma(t)$ be a continuous curve. For $f$ in $\delta$, set

$$
\begin{aligned}
& \mathcal{H}^{*} f(x)=\sup _{0<\varepsilon<N<\infty}\left|\int_{\varepsilon<|t|<N} f(x-\gamma(t)) \frac{d t}{t}\right|, \text { and } \\
& \mathcal{K}_{1}^{*} f(x)=\sup _{\substack{\varepsilon \\
0<\varepsilon<1}}\left|\int_{\varepsilon<|t|<1} f(x-\gamma(t)) \frac{d t}{t}\right| .
\end{aligned}
$$

Then we have

THEOREM 6. (A) Let $\gamma(t)$ be a two-sided homogeneous curve, then

$$
\|\mathcal{H C} * f\|_{2}<c\|f\|_{2} \text {. }
$$

(B) Let $\gamma(t)$ be in $C^{\infty}$ in $[-1,1]$. Assume an initial segment of $\gamma(t)$ lies in the subspace spanned by $\left\{\gamma^{(j)}(0)\right\}_{j=1}^{\infty}$. Then

$$
\left\|\mathcal{H}_{1}^{*} f\right\|_{2} \leqslant c\|f\|_{2} \text {. }
$$

Proof of Theorem 6. Consider first part (A). We shall assume that $\gamma(t)$ lies in no proper subspace. The removal of this assumption is as in the proof of Theorem 3. For $f$ in $\mathcal{S}$, we set

$$
\mathcal{F}_{\lambda} f(x)=\int_{|t|>\lambda} f(x-\gamma(t)) \frac{d t}{t}
$$

Clearly

$$
\widehat{\mathcal{F}} \widehat{\lambda f(\xi)}=m_{1}\left(\delta_{\lambda}^{*} \xi\right) \hat{f}(\xi)
$$

with

$$
m_{1}(\xi)=\int_{|t|>1} \exp \{-2 \pi i \xi \cdot \gamma(t)\} \frac{d t}{t} .
$$

We let $\eta(\xi)$ be in $C_{0}^{\infty}$ with $\eta(\xi)$ identically one for $\xi$ near 0 .

We shall define a $g$-function in terms of an operator $\mathcal{R}_{\lambda}$ given by the formula

$$
\widehat{\Re_{\lambda} f}(\xi)=\left(1-\eta\left(\delta_{\lambda}^{*} \xi\right)\right) m_{1}\left(\delta_{\lambda}^{*} \xi\right) \hat{f}(\xi) .
$$

(The fact that $\widehat{\Re_{\lambda} f}$ vanishes near $\xi=0$ will be especially convenient later when we deal with the $L^{p}$ theory.) We set 


$$
g^{2}(f)(x)=\int_{0}^{\infty} \frac{d \lambda}{\lambda}\left|\Re_{\lambda} f(x)\right|^{2} .
$$

Using arguments similar to the proof of Theorems 3 and 5, we see that

$$
\|g(f)\|_{2} \leqslant c\|f\|_{2} \text {. }
$$

Now

$$
\Re_{\lambda} f=\mathcal{H}_{\lambda} f-\check{\eta_{\lambda}} * \mathcal{H} f+E_{\lambda} * f
$$

where

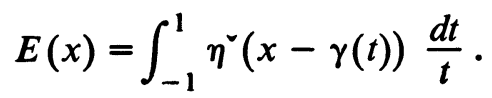

$\left(\eta_{\lambda}^{\circ}(x)=\lambda^{-a} \eta^{\nu}\left(\delta_{\lambda}^{-1} x\right)\right.$, and $E_{\lambda}(x)=\lambda^{-a} E\left(\delta_{\lambda}^{-1} x\right)$.) Now

$$
\begin{aligned}
|E(x)| & \leqslant \int_{-1}^{1}\left|\eta^{\sim}(x-\gamma(t))-\eta^{\circ}(x)\right| \frac{d t}{t} \\
& \leqslant \frac{c_{\alpha}}{\left(1+|x|^{\alpha}\right)} \quad \text { for any } \alpha .
\end{aligned}
$$

The same estimate is true for $\eta^{\circ}$. Thus by (3-12), (3-13), and Theorem 3 (arguing as in the proof of Theorem 4), we obtain

$$
\left\|\sup _{h>0}\left|\frac{1}{h} \int_{0}^{h} \int_{|t|>\lambda} f(x-\gamma(t)) \frac{d t}{t} d \lambda\right|\right\|_{2} \leqslant c\|f\|_{2} .
$$

So by Theorem 3

$$
\left\|\sup _{h>0}\left|\frac{1}{h} \int_{0}^{h} \int_{|t|<\lambda} f(x-\gamma(t)) \frac{d t}{t} d \lambda\right|\right\|_{2} \leqslant c\|f\|_{2} ;
$$

or interchanging the order of integration

$$
\left\|\sup _{h>0}\left|\int_{-h}^{h} f(x-\gamma(t))\left(\frac{1}{h} \int_{|t|}^{h} d \lambda\right) \frac{d t}{t}\right|\right\|_{2} \leqslant c\|f\|_{2} .
$$

Hence by Theorem 4,

$$
\left\|\sup _{h>0}\left|\int_{-h}^{h} f(x-\gamma(t)) \frac{d t}{t}\right|\right\|_{2} \leqslant c\|f\|_{2} .
$$

This implies Theorem 6, part (A).

To prove part (B), we can assume $\gamma(t)$ is of standard type. Then we make the following modifications: We set

$$
\mathcal{H}_{\lambda} f(x)=\int_{\lambda<|t|<1} f(x-\gamma(t)) \frac{d t}{t}
$$

Then

$$
\widehat{\mathcal{F}_{\lambda} f}(\xi)=m_{\lambda}(\xi) \hat{f}(\xi)
$$

where 


$$
m_{\lambda}(\xi)=\int_{\lambda<|t|<1} \exp (-2 \pi i \xi \cdot \gamma(t)) \frac{d t}{t} .
$$

We define $\Re_{\lambda} f$ by

$$
\widehat{\Re_{\lambda} f}(\xi)=\left(1-\eta\left(\delta_{\lambda} \xi\right)\right) m_{\lambda}(\xi) \hat{f}(\xi)
$$

where $\delta_{\lambda}$ is the dilation group corresponding to $\gamma($ see $\S 1)$. We set

$$
g^{2}(f)(x)=\int_{0}^{1} \frac{d \lambda}{\lambda}\left|\Re_{\lambda} f(x)\right|^{2}
$$

One proves

$$
\|g(f)\|_{2} \leqslant c\|f\|_{2}
$$

using modifications already used in the proofs of part (B) of Theorems 3 and 4. The remainder of the argument follows by a simple modification of that proving part (A). (However instead of dealing with $E_{\lambda}$ as above we must observe that

$$
\begin{aligned}
& \quad \sup _{0<\lambda<1}\left|\left\{\frac{1}{\lambda^{a}} \int_{-\lambda}^{\lambda} \eta^{-}\left[\delta_{\lambda}^{-1}(x-\gamma(t))\right] \frac{d t}{t}\right\} * f\right| \\
& =\sup _{0<\lambda<1}\left|\left\{\frac{1}{\lambda^{a}} \int_{-1}^{1} \eta^{-}\left[\delta_{\lambda}^{-1} x-\delta_{\lambda}^{-1} \gamma(\lambda t)\right] \frac{d t}{t}\right\} * f\right| \\
& =\sup _{0<\lambda<1}\left|\left\{\frac{1}{\lambda^{a}} \int_{-1}^{1} \eta^{-}\left[\delta_{\lambda}^{-1} x-\delta_{\lambda}^{-1} \gamma(\lambda t)\right]-\eta^{-}\left[\delta_{\lambda}^{-1} x\right] \frac{d t}{t}\right\} * f\right| \\
& <c_{\alpha} \sup _{\lambda>0} \frac{1}{\lambda^{a}} \frac{1}{\left[1+\left|\delta_{\lambda}^{-1} x\right|\right]^{\alpha}} *|f|
\end{aligned}
$$

for any $\alpha>0$; and this term is bounded in $L^{2}$ by the argument which proves $\left(3-5^{\prime}\right)$.)

(d) Maximal spherical averages. Here, for $f$ in $\delta$, we put

$$
M_{\imath} f(x)=\int_{|y|=1} f(x-t y) d \sigma(y)
$$

where $d \sigma$ is the rotationally invariant measure of total mass 1 on the unit sphere. We put

$$
\mathfrak{N} f(x)=\sup _{t>0}\left|M_{t} f(x)\right|
$$

We then have

THEOREM 7. If $n \geqslant 3$,

$$
\|\mathfrak{N} f\|_{2} \leqslant c\|f\|_{2}
$$

Proof. It is well known that

$$
\widehat{M_{t} f}(\xi)=m(t \xi) \hat{f}(\xi)
$$

where 


$$
m(\xi)=2^{n / 2-1} \Gamma(n / 2)(2 \pi|\xi|)^{-n / 2+1} J_{n / 2-1}(2 \pi|\xi|) .^{7}
$$

To prove Theorem 7, we shall need to consider additional operators $M_{t}^{\alpha}$. For $f$ in $\mathfrak{S}$, we set

$$
\widehat{M_{t}^{\alpha} f}(\xi)=m_{\alpha}(t \xi) \hat{f}(\xi)
$$

where

$$
m_{\alpha}(\xi)=2^{n / 2+\alpha-1} \Gamma(n / 2+\alpha)(2 \pi|\xi|)^{-n / 2-\alpha+1} J_{n / 2-1+\alpha}(2 \pi|\xi|) .
$$

To prove Theorem 7 , we would only need to consider real $\alpha$ 's, but we shall need to consider complex $\alpha$ 's when we deal with $L^{p}$ versions of Theorem 7 .

We shall not prove Theorem 7 directly by the use of $g$-functions, but instead we shall use $g$-functions to discuss $\mathfrak{N}^{*} f$, where

$$
\Re^{*} f(x)=\sup _{t>0}\left\{\frac{1}{t} \int_{0}^{t}\left|M_{s}^{\alpha} f(x)\right|^{2} d s\right\}^{1 / 2} \text {. }
$$

THEOREM 8. For $\alpha>1 / 2-n / 2$

$$
\|\mathfrak{T} * f\|_{2} \leqslant c_{\alpha}\|f\|_{2} \text {. }
$$

Moreover if $\alpha$ is in a compact subinterval of $(1 / n-n / 2, \infty), c_{\alpha}$ is bounded.

We shall then use Theorem 8 to prove the following strong form of Theorem 7:

THEOREM 9. Let

$$
\mathscr{T}^{\alpha} f(x)=\sup _{t>0}\left|M_{t}^{\alpha} f(x)\right|
$$

If $R \alpha>1-n / 2$,

$$
\left\|\Re^{\alpha} f\right\|_{2} \leqslant c_{\alpha}\|f\|_{2} \text {. }
$$

Moreover, if $R \alpha$ is in a compact subinterval of $(1 / 2-n / 2, \infty)$, then

$$
\log \left|c_{\alpha}\right| \leqslant c[1+|\alpha| \log |\alpha|] .
$$

The fact that Theorem 8 implies Theorem 9 is a consequence of the identity

$$
M_{t}^{\alpha} f(x)=c(n, \alpha) \int_{0}^{1} M_{s t}^{\alpha^{\prime}} f(x)\left(1-s^{2}\right)^{\alpha-\alpha^{\prime}-1} s^{n+2 \alpha^{\prime}-1} d s
$$

whenever $R \alpha>R \alpha^{\prime}$ and $R \alpha^{\prime}>-n / 2$, with

$$
c(n, \alpha)=2 \Gamma(n / 2+\alpha) /\left(\Gamma\left(n / 2+\alpha^{\prime}\right) \Gamma\left(\alpha-\alpha^{\prime}\right)\right) .
$$

(Upon taking Fourier transforms (3-14) is seen to be equivalent to the identity

$$
\begin{aligned}
& (2 \pi t|\xi|)^{-n / 2-\alpha+1} J_{n / 2-1+\alpha}(2 \pi t|\xi|)=\frac{2^{\alpha^{\prime}-\alpha+1}}{\Gamma\left(\alpha-\alpha^{\prime}\right)} \\
& \cdot \int_{0}^{1}(2 \pi s t|\xi|)^{-n / 2-\alpha^{\prime}+1} J_{n / 2-1+\alpha^{\prime}}(2 \pi s t|\xi|)\left(1-s^{2}\right)^{\alpha-\alpha^{\prime}-1} s^{n+2 \alpha^{\prime}-1} d s,
\end{aligned}
$$

which is essentially equation 5, p. 46, vol. 2 of Erdelyi et al. [1953].) Now an

\footnotetext{
${ }^{7}$ See e.g. Stein and Weiss [1971, pp. 154-155].
} 
application of Schwarz's inequality in (3-14) shows that Theorem 8 implies Theorem 9.

Theorem 8 in turn follows easily from the $L^{2}$ boundedness of an appropriate $g$-function. As usual we let $\phi$ be a $C_{0}^{\infty}$ function with $\hat{\phi}(0)=1$. We set $\phi_{t}(x)=t^{-n} \phi(x / t)$, and

$$
g_{\alpha}(f)(x)=\left\{\int_{0}^{\infty}\left|M_{t}^{\alpha} f(x)-f * \phi_{t}(x)\right|^{2} \frac{d t}{t}\right\}^{1 / 2}
$$

We then have

THEOREM 10. If $\alpha$ is real and $\alpha>1 / 2-n / 2$,

$$
\left\|g_{\alpha}(f)\right\|_{2} \leqslant c_{\alpha}\|f\|_{2} \text {. }
$$

Moreover $c_{\alpha}$ is bounded if $\alpha$ is restricted to lie in a compact subinterval of $(1 / 2-n / 2, \infty)$.

Proof of Theorem 10. Using Parseval's theorem as in the proof of Theorem 5, the proof of Theorem 10 comes down to the estimate

$$
\int_{0}^{\infty}\left|m^{\alpha}(t \xi)-\hat{\phi}(t \xi)\right|^{2} \frac{d t}{t} \leqslant c_{\alpha}
$$

for $|\xi|=1$.

Since $m^{\alpha}(0)=\hat{\phi}(0)=1$, the portion of the integral $t \leqslant 1$ in (3-15) is easily seen to be bounded. To deal with the contribution for large $t$, we note

$$
\left|m_{\alpha}(t \xi)\right| \leqslant c_{\alpha} t^{-n / 2-\alpha+1 / 2}
$$

where $c_{\alpha}$ is bounded if $\alpha$ is restricted to $\alpha$ compact set.

4. A priori $L^{p}$ estimates for the Hilbert transform. Our main goal here is the following theorem: $\delta$,

THEOREM 11. (A) Let $\gamma(t)$ be a two-sided homogeneous curve. Then for $f$ in

$$
\left\|\mathcal{H}_{\varepsilon, N} f\right\|_{p} \leqslant C(p, \gamma)\|f\|_{p}, \quad 1<p<\infty .
$$

Moreover $\mathcal{H}_{\varepsilon, N}$ fonverges in $L^{p}$ to $\mathcal{H} f$, as $\varepsilon \rightarrow 0, N \rightarrow \infty$, and

$$
\|\mathcal{H} f\|_{p}<C(p, \gamma)\|f\|_{p} .
$$

(B) Let $\gamma(t)$ be a $C^{\infty}$ curve on $[-1,1]$. Assume $\gamma(t)$ lies in the subspace spanned by $\left\{\gamma^{(j)}(0)\right\}_{j=1}^{\infty}{ }^{8}$ Then conclusions analogous to those of part (A) hold for $\mathcal{H}_{\varepsilon, 1}$.

Proof of PART (A). It suffices to prove (i). We assume first that $\gamma(t)$ does not lie in a proper subspace of $R^{n}$. We consider an analytic family of operators $T_{2} f$ defined by

$$
\widehat{T_{z} f}(\xi)=\left\{\rho^{*}(\xi)\right\}^{z} m_{z}(\xi) \hat{f}(\xi),
$$

where

\footnotetext{
${ }^{8}$ It suffices of course that this condition on $\gamma(t)$ be satisfied in any neighborhood of $t=0$.
} 


$$
m_{z}(\xi)=\int_{\varepsilon<|t|<N} \exp \{-2 \pi i \xi \cdot \gamma(t)\}|t|^{2} \frac{d t}{t} .
$$

Our object is the inequality

$$
\left\|T_{0} f\right\|_{p} \leqslant A_{p}\|f\|_{p}
$$

By duality, we may assume $1<p \leqslant 2$. Then by the analytic interpolation theorem, Stein and Weiss [1971, p. 205], it suffices to prove the following two lemmas:

LEMMA 4-2. There is a positive $\alpha$ such that

$$
\left\|T_{z} f\right\|_{2} \leqslant C(z)\|f\|_{2}
$$

for $-\alpha<R z<\alpha$, where $C(z)$ grows at most polynomially in $|z|$.

LEMMA 4-3. For an arbitrarily small positive number $\eta$, and suitable $\beta$

$$
\left\|T_{z} f\right\|_{p} \leqslant C(z, p)\|f\|_{p}, \quad 1<p<\infty,
$$

for $-\beta \leq R z<-\eta$. Moreover for $\eta$ fixed, $C(z)$ grows at most as fast as a polynomial in $|z|$.

(There is one slight technical point to be mentioned. We prove Lemmas 4-2 and 4-3 for $f$ simple. This will give (4-1) only for $f$ simple. However for any $f$ in $\mathcal{S}$, we can easily find simple functions $f_{n}$ such that $f_{n} \rightarrow f$ in $L^{p}$ and $\mathcal{H}_{\varepsilon, N} f_{n} \rightarrow \mathcal{H}_{\varepsilon, N} f$ in $L^{p}$, which will give (4-1) for $f$ in $\delta$.)

The proof of Lemma 4-2 is essentially the same as the proof of Theorem 3.

To prove Lemma 4-3 we need an additional lemma.

LEMMA 4-4. For $-a<R z<0$

$$
\left\{\rho^{*}(\xi)\right\}^{z}=\hat{h}_{z}(\xi)
$$

where $h_{z}(x)$ is a locally integrable function, $C^{\infty}$ away from the origin satisfying

$$
h_{z}\left(\delta_{\lambda} x\right)=\lambda^{-a-z} h_{z}(x), \quad \lambda>0, \quad x \neq 0 .
$$

Moreover each derivative of $h_{z}(x)$ is bounded by a polynomial in $|z|$, if $\rho(x)=1$. (Here $a=\operatorname{tr} A$, and the Fourier transform is to be taken in the sense of distributions.)

Proof of Lemma 4-4. Let $\phi$ be in $C_{0}^{\infty}$ with $\phi(\xi)=1$ in a neighborhood of 0 . Then

$$
\begin{aligned}
{\left[\rho^{*}(\xi)\right]^{2} } & =\phi(\xi)\left[\rho^{*}(\xi)\right]^{2}+(1-\phi(\xi))\left[\rho^{*}(\xi)\right]^{2} \\
& =r_{1}(\xi)+r_{2}(\xi) .
\end{aligned}
$$

$r_{1}(\xi)$ has compact support and is thus the Fourier transform of a $C^{\infty}$ function. But if $b_{1}, b_{2}, \ldots, b_{n}$ are any nonnegative integers, and $\beta$ is a sufficiently large positive integer

$$
\Delta^{\beta}\left\{\xi_{1}^{b_{1}} \cdots \xi_{n}^{b_{n}} r_{2}(\xi)\right\} \in L^{1} .
$$

(See Proposition 1-9 (vi).) Thus 


$$
|x|^{2 \beta} \frac{\partial^{b_{1}+\cdots+b_{n}} \check{r}_{2}(x)}{\partial x_{1}^{b_{1}} \cdots \partial x_{n}^{b_{n}}}
$$

is continuous. We define $h_{z}(x)$ to be the distribution such that

$$
\hat{h}_{z}(\xi)=\left[\rho^{*}(\xi)\right]^{z} \text {. }
$$

Then the above discussion shows

$$
h_{z}(x)=u_{z}(x)+v_{z}(x)+w_{z}(x)
$$

with $u_{z}(x)$ in $C^{\infty}, v_{z}(x)$ a linear combination of $\delta$-functions and derivatives of $\delta$-functions supported at the origin, and $w_{z}(x)$ is in $C^{\infty}\left(R^{n}-0\right)$ and is locally integrable.

Now

$$
\rho^{*}\left(\delta_{\lambda}^{*} \xi\right)^{z}=\lambda^{z} \rho^{*}(\xi), \quad \lambda>0 .
$$

This implies

$$
h_{z}\left(\delta_{\lambda} x\right)=\lambda^{-a-z} h_{z}(x) \quad \text { (as distributions). }
$$

Then we see that

$$
u_{z}\left(\delta_{\lambda} x\right)+w_{z}\left(\delta_{\lambda} x\right)=\lambda^{-a-z}\left(u_{z}(x)+w_{z}(x)\right)
$$

by operating on functions vanishing near the origin. Thus

$$
v_{z}\left(\delta_{\lambda} x\right)=\lambda^{-a-z} v_{z}(x) \text {. }
$$

Since $R z>0$, and $v_{z}$ is a linear combination of $\delta$-functions and their derivatives then $v_{z} \equiv 0$. We turn now to the proof of Lemma 4-3. By Lemma 4-4, we see that

$$
T_{z} f(x)=K_{z} * f
$$

where

$$
K_{z}(x)=\int_{\varepsilon<|t|<N} h_{z}(x-\gamma(t))|t|^{z} \frac{d t}{t}
$$

for $f$ in $\mathcal{S}$. It follows easily that (4-5) holds when $f$ is simple.

We shall now show that for sufficiently large constants $C$ and $C_{1}(z)$

$$
\int_{\rho(x)>C \rho(y)}\left|K_{z}(x-y)-K_{z}(x)\right| d x \leqslant C_{1}(z) .
$$

If $-\alpha \leqslant R z \leqslant-\eta<0$. Moreover $C_{1}(z)$ can be taken to be at most of polynomial growth in $|z|$. (4-7) will establish Lemma 4-3 by virtue of Theorem 4.1 of Rivière [1971]. (The regular Vitali family $\left(U_{\alpha}, \Phi_{\alpha}\right)$ is given by

$$
U_{\alpha}=\{x \mid \rho(x)<\alpha\} \text {. }
$$

$\Phi_{\alpha}=C_{1} \alpha$ provided $C_{1} \geqslant 2 C, C$ being the constant of Proposition 1-9 (ii).)

To prove (4-7), we note first that a change of variables $x=\delta_{\rho(y)} x^{\prime}$ and $t=s \rho(y)$ allows us to assume without loss of generality that $\rho(y)=1$. By the homogeneity of $h$ and the smoothness of $h$ away from 0 , we have

$$
\left|h_{z}(x-\omega)-h_{z}(x)\right| \leqslant C(z) \frac{|\omega|}{\{\rho(x)\}^{a+R z+\mu}}
$$


for some $\mu>0$, provided $|\omega| /|x|$ is sufficiently small. We then set

$$
K_{z}(x)=K_{z}^{1}(x)+K_{z}^{2}(x),
$$

where

$$
K_{z}^{1}(x)=\int_{\varepsilon<|t|<1} h_{z}(x-\gamma(t))|t|^{z} \frac{d t}{t}
$$

Then

$$
\left|K_{z}^{1}(x)\right|<\int_{\varepsilon<|t|<1}\left|h_{z}(x-\gamma(t))-h_{z}(x)\right||t|^{2} \frac{d t}{t} .
$$

So using (4-8) and Fubini's theorem,

$$
\begin{aligned}
& \int_{\rho(x)>C}\left|K_{z}^{1}(x)\right| d x \leqslant \int_{t<1} t^{R_{z}-1} \int_{\rho(x)>C}\left|h_{z}(x-\gamma(t))-h_{z}(x)\right| d x d t \\
& <C(z) \int_{|t|<1} t^{R_{z}-1}|\gamma(t)| \int_{\rho(x)>C}\{\rho(x)\}^{-a-R z-\mu} d x d t \leqslant C_{1}(z)
\end{aligned}
$$

if $R z$ is sufficiently close to zero by (1.6) and Proposition 1-9 (v).

A similar argument shows

$$
\int_{\rho(x)>C}\left|K_{z}^{1}(x-y)\right| d x \leqslant C(z) .
$$

Finally

$$
\begin{aligned}
\int_{\rho(x)>C} & \left|K_{z}^{2}(x-y)-K_{z}^{2}(x)\right| d x \\
& <\int_{|t|>1} t^{-1+R z} \int_{\rho(x)>C}\left|h_{z}(x-\gamma(t)-y)-h_{z}(x-\gamma(t))\right| d x d t .
\end{aligned}
$$

We divide the inner integral above according to whether $\rho(x-\gamma(t))>C_{2}$ or $\rho(x-\gamma(t))<C_{2}$ for an appropriate large constant $C_{2}$. The portion where $\rho(x-\gamma(t))>C_{2}$ is uniformly bounded by (4-8). The portion $\{x \mid \rho(x-\gamma(t))$ $\left.\leqslant C_{2}\right\}$ is uniformly bounded since $h_{z}$ is locally integrable.

We thus have

$$
\int_{\rho(x)>C}\left|K_{z}^{2}(x-y)-K_{2}^{2}(x)\right| d x \leqslant C_{3}(z) .
$$

Now (4-9)-(4-11) give (4-7).

As usual there is little trouble in removing the assumption that $\gamma(t)$ does not lie in a proper subspace of $R^{n}$.

There is little extra difficulty in proving part (B). One first notes that it suffices to consider the case that $\gamma(t)$ is of standard type. We then define $T_{z}$ as in the proof of part (A), where $\rho$ is the norm function associated to a curve of standard type as in $\S 1$. (Except, of course, the integral defining $m_{z}(\xi)$ extends only from $\varepsilon$ to 1.) The proof of the analogue of Lemma 4-2 follows by the same reasoning as that of Theorem 3 part (B). To prove the analogue of Lemma 4-3, we shall verify (4-7) with 


$$
K_{z}(x)=\int_{\varepsilon<|t|<1} h_{z}(x-\gamma(t))|t|^{2} \frac{d t}{t}
$$

where $R z$ is slightly negative and $h_{z}$ is the function of Lemma 4-4. The argument above giving (4-9) now yields

$$
\int_{\rho(x)>C}\left|K_{z}(x)\right| d x<C(z)
$$

if $C$ is sufficiently large. Thus if $\rho(y)$ is bounded away from zero (4-7) is easily verified. We therefore consider the case that $\rho(y)$ is small. We set $x=\delta_{\rho(y)} x^{\prime}$ and $t=s \rho(y)$ (in the integral defining $K_{z}$ ) to obtain

$$
\begin{aligned}
& \int_{\rho(x)>C \rho(y)}\left|K_{z}(x-y)-K_{z}(x)\right| d x \\
&=\left.\int_{\rho\left(x^{\prime}\right)>C}\left|\int_{\varepsilon / \rho(y)<s<1 / \rho(y)}\right| s\right|^{2}\left\{h_{z}\left[x^{\prime}-y-\delta_{1 / \rho(y)} \gamma(s \rho(y))\right]\right. \\
&\left.\quad-h_{z}\left[x^{\prime}-\delta_{1 / \rho(y)} \gamma(s \rho(y))\right]\right\} \frac{d s}{s} \mid d x^{\prime} .
\end{aligned}
$$

We next divide the $s$ integral according to where $s \leqslant 1$ and where $s \geqslant 1$. When $s<1$ we utilize the argument establishing (4.9). (For this portion of the integral we use the fact that $\left|\delta_{1 / \rho(y)} \gamma(s \rho(y))\right| \leqslant C|\gamma(s)|$. Finally, the contribution from $s \geqslant 1$ is handled by the argument proving (4-10).)

5. A priori $L^{p}$ estimates for the maximal function. Here we extend the results of $\S 3(\mathrm{~b})$ to $L^{p}$ spaces with $1<p \leq \infty$. We prove the following theorem:

THEOREM 12. (A) If $\gamma(t)$ is a homogeneous curve and $f$ is in $\delta$ then

$$
\|\Re f\|_{p} \leqslant C_{p}\|f\|_{p}, \quad 1<p<\infty .
$$

(B) Let $\gamma(t)$ be in $C^{\infty}$. Assume $\gamma(t)$ lies in the subspace spanned by $\left\{\gamma^{(j)}(0)\right\}_{j=1}^{\infty}$. Then for $f$ in $\delta$,

$$
\left\|\Re_{1} f\right\|_{p} \leqslant C_{p}\|f\|_{p}, \quad 1<p \leqslant \infty .
$$

Proof. By the Marcinkiewicz interpolation theorem and the fact that the result is trivial when $p=\infty$ Stein-Weiss [1971, p. 184] we only need deal with the case $1<p<2$.

We consider first part (A). As usual we first assume $\gamma(t)$ does not lie in a proper subspace of $R^{n}$. (This restriction can be dropped by an argument used in the proof of Theorem 3.) As in $\$ 3(b)$, we set

$$
\widehat{N_{h} f}(x)=\frac{1}{h} \int_{h}^{2 h} f(x-\gamma(t)) d t .
$$

Using part of the argument of the proof of Theorem 4, we see that it suffices to show

$$
\left\|\sup _{h>0} \frac{1}{h} \int_{0}^{h} N_{s} f(x) d s\right\|_{p} \leqslant C_{p}\|f\|_{P}
$$


for $f \geqslant 0$ in $\delta$. As pointed out in $\$ 3(\mathrm{~b})$,

$$
\widehat{N_{h} f}(\xi)=\hat{f}(\xi) n\left(\delta_{h}^{*} \xi\right)
$$

where

$$
n(\xi)=\int_{1}^{2} \exp (-2 \pi i \xi \cdot \gamma(t)) d t .
$$
by

Let $\eta(\xi)$ be in $C_{0}^{\infty}$ with $\eta(\xi)=1$ for $\xi$ near 0 . We then define $N_{h}^{z} f$ for $f$ in $\delta$, $\widehat{N_{h}^{z} f}(\xi)=\eta\left(\delta_{h}^{*} \xi\right) n\left(\delta_{h}^{*} \xi\right) \hat{f}(\xi)+\rho^{* z}\left(\delta_{h}^{*} \xi\right)\left(1-\eta\left(\delta_{h}^{*} \xi\right)\right) n\left(\delta_{h}^{*} \xi\right) \hat{f}(\xi)$.

Further, we let $\phi(x)$ be in $C_{0}^{\infty}$ with $\hat{\phi}(0)=1$, and set

$$
g_{z}(f)(x)=\left\{\int_{0}^{\infty} \frac{d h}{h}\left|N_{h}^{z} f(x)-\phi_{h} * f(x)\right|^{2}\right\}^{1 / 2} .
$$

Then an argument analogous to the proof of Thoerem 5 yields

$$
\left\|g_{z}(f)\right\|_{2} \leqslant C(z)\|f\|_{2}
$$

for $R z<\sigma$, where $\sigma$ is as in (2.1). Moreover $C(z)$ grows only as fast as a polynomial in $|z|$ if $R(z)$ lies in a compact subinterval of $(-\infty, \sigma)$.

The argument of Theorem 4 then gives

LEMMA 5-4. If $-\sigma / 2 \leqslant R z \leqslant \sigma / 2$ and $f$ in $\delta$

$$
\left\|\frac{1}{h(x)} \int_{0}^{h(x)} N_{s}^{z} f(x) d s\right\|_{2} \leqslant C(z)\|f\|_{2}
$$

for any positive measurable function $h(x)$. Moreover, we can take $C(z)$ so that it grows at most polynomially in $|z|$.

We shall also show

LEMMA 5-5. There is $a \sigma_{2}>0$ such that if $0<\sigma_{1}<\sigma_{2}$ and $-\sigma_{2}<R(z)<$ $-\sigma_{1}$

$$
\left\|\frac{1}{h(x)} \int_{0}^{h(x)} N_{s}^{z} f(x) d s\right\|_{p} \leqslant C_{p}(z)\|f\|_{p}, \quad 1<p \leqslant \infty,
$$

for every positive measurable function $h(x)$. Moreover if $\sigma_{1}$ is fixed $C_{p}(z)$ may be taken so that $\left|C_{p}(z)\right|$ grows at most like a polynomial in $|z|$.

Lemmas 5-4 and 5-5 will imply (5-1) (and hence part (A) of Theorem 12) by analytic interpolation provided we overcome the following two technical points:

(a) Lemmas 5-4 and 5-5 hold only for $f$ in $\delta$ and not for simple functions as the interpolation theorem requires.

(b) The transformation

$$
f \rightarrow \frac{1}{h(x)} \int_{0}^{h(x)} N_{s}^{z}(f)(x) d s
$$


must be shown to be analytic in the sense required by the analytic interpolation theorem.

We deal with the first difficulty by letting $\Phi_{\varepsilon}$ be an approximate identity and considering

$$
f \rightarrow \frac{1}{h(x)} \int_{0}^{h(x)} N_{s}^{z}\left(f * \Phi_{\varepsilon}\right)(x) d s .
$$

It is easy to deal with (b) if $h(x)$ takes only a finite set of values. This clearly suffices for $f$ in $\mathcal{S}$.

To prove Lemma 5-5 we need the following lemma due to F. Zo.

LeMma 5-6. Let $K_{h}(x)$ be in $L^{1}$ for $h>0$. Assume

(i) $\int\left|K_{h}(x)\right| d x \leqslant C<\infty$ for $h>0$, and

(ii) $\int_{\rho(x)>C \rho(y)} \sup _{h>0}\left|K_{h}(x-y)-K_{h}(x)\right| d x \leqslant C$. Set

$$
\mathscr{K} f(x)=\sup _{h>0}\left|K_{h} * f(x)\right|
$$

Then

$$
\|\mathcal{H} f\|_{p} \leqslant A_{p}\|f\|_{p}, \quad 1<p \leqslant \infty .
$$

Also $A_{p}$ depends only on $C$.

(Zo's lemma actually also includes the weak type 1,1 conclusion, though we shall not use it here. See Zo [1975] for the general case or Zo [1977], where however only the case $\rho(x)=|x|$ is considered.)

Lemmas 5-6 and 5-4 will imply Lemma 5-5 modulo some trivial terms. The crux of the argument is contained in the following lemma:

LEMMA 5-7. Let $\psi(t)$ be a $C_{0}^{\infty}$ function defined for $0 \leqslant t<\infty$, vanishing near 0 . Let $\lambda$ be a positive number with $\lambda<a$, but sufficiently close to a. Define

$$
K(x)=\psi(\rho(x)) \int_{1}^{2} \frac{d t}{\{\rho(x-\gamma(t))\}^{\lambda}} .
$$

Then $K_{h}(x)=h^{-a} K\left(\delta_{h}^{-1} x\right)$ satisfies the hypothesis of Lemma 5-6.

Proof of Lemma 5-7. Condition (i) is trivial. So we need to prove condition (ii). By homogeneity we may assume $\rho(y)=1$. Now

$$
\begin{aligned}
K_{h}(x) & =h^{-a} \psi\left(\frac{1}{h} \rho(x)\right) \int_{1}^{2} \frac{d t}{\left\{\rho\left(\delta_{h}^{-1} x-\gamma(t)\right)\right\}^{\lambda}} \\
& =h^{\lambda-a-1} \psi\left(\frac{1}{h} \rho(x)\right) \int_{h}^{2 h} \frac{d t}{\{\rho(x-\gamma(t))\}^{\lambda}} .
\end{aligned}
$$




$$
\begin{aligned}
&\left|K_{h}(x-y)-K_{h}(x)\right| \leqslant h^{\lambda-a-1}\left\{\left|\psi\left(\frac{1}{h} \rho(x-y)\right)-\psi\left(\frac{1}{h} \rho(x)\right)\right|\right\} \\
& \cdot \int_{h}^{2 h} \frac{d t}{[\rho(x-y-\gamma(t))]^{\lambda}} \\
&+h^{\lambda-a-1} \psi\left(\frac{\rho(x)}{h}\right) \int_{h}^{2 h}\left|\frac{1}{\rho^{\lambda}(x-y-\gamma(t))}-\frac{1}{\rho^{\lambda}(x-\gamma(t))}\right| d t .
\end{aligned}
$$

Since $\psi(t)$ vanishes near 0 and $\infty, \rho(x)$ must be between two multiples of $h$ or $K_{h}(x-y)-K_{n}(x)=0$ (at least if $\rho(x)$ is large and $\rho(y)=1$ ). Thus for $\rho(y)=1$ and $\rho(x)$ large,

$$
\begin{aligned}
\sup _{h>0}\left|K_{h}(x-y)-K_{h}(x)\right| \\
\quad<C\{\rho(x)\}^{\lambda-a-2}|\rho(x-y)-\rho(x)| \int_{C_{1} \rho(x)}^{C_{2} \rho(x)} \frac{d t}{\{\rho(x-y-\gamma(t))\}^{\lambda}} \\
+C\{\rho(x)\}^{\lambda-a-1} \int_{C_{1} \rho(x)}^{C_{2} \rho(x)}\left|\frac{1}{\rho^{\lambda}(x-y-\gamma(t))}-\frac{1}{\rho^{\lambda}(x-\gamma(t))}\right| d t .
\end{aligned}
$$

Thus for sufficiently large $C$

$$
\begin{aligned}
& \int_{\rho(x)>C} \sup _{h>0}\left|K_{h}(x-y)-K_{h}(x)\right| d x \\
& <C \int_{t>C_{3}} t^{\lambda-a-1} \int_{C_{4} t<\rho(x)<C_{5} t} \frac{1}{\rho(x)} \frac{|\rho(x-y)-\rho(x)|}{\{\rho(x-y-\gamma(t))\}^{\lambda}} d x d t \\
& +C \int_{t>C_{3}} t^{\lambda-1-a} \int_{C_{4} t<\rho(x)<C_{5} t}\left|\frac{1}{\rho^{\lambda}(x-y-\gamma(t))}-\frac{1}{\rho^{\lambda}(x-\gamma(t))}\right| d x d t .
\end{aligned}
$$

Hence to prove Lemma 5-6, it suffices to show the following two facts:

$$
\int_{\rho(x)>C_{6}} \frac{|\rho(x-y)-\rho(x)|}{\rho(x) \rho^{\lambda}(x-u)} d x<C
$$

for sufficiently large $C$ (for all $u$ ) and

$$
\int\left|\frac{1}{\rho^{\lambda}(x-y-u)}-\frac{1}{\rho^{\lambda}(x-u)}\right| d x<C,
$$

for sufficiently large $C$.

Since $\rho^{-\lambda}(x)$ is locally integrable, (5-9) follows from the following inequality:

If $\omega$ is sufficiently large and $\rho(y)=1$,

$$
\left|\rho^{\alpha}(\omega-y)-\rho^{\alpha}(\omega)\right| \leqslant C \rho^{\alpha-\mu}(\omega)
$$

for any $\alpha$ and some positive $\mu$, and this follows from Proposition 1-9 (vi).

Using the above inequality again we see that to prove (5-8) it suffices to show that 


$$
\int_{\rho(x)>C}\{\rho(x)\}^{-\varepsilon} \rho^{-\lambda}(x-u) d x \leqslant C,
$$

for a fixed $\varepsilon>0$. We may assume $\rho(u) \geqslant 1$ since if $\rho(u)<1$, the inequality is trivial. We now break up the region of integration into parts $\rho(x-u)<$ $\rho(x) / 10$, and $\rho(x-u) \geqslant \rho(x) / 10$. The second integral is majorized by

$$
C \int_{\rho(x)>C}[\rho(x)]^{-\varepsilon-\lambda} d x \leqslant C .
$$

In the first region, $\rho(x) \approx \rho(u)$, so the corresponding integral is majorized by

$$
\{\rho(u)\}^{-\varepsilon} \int_{\rho(x)<C \rho(u)} \rho^{-\lambda}(x) d x \leqslant C(\rho(u))^{-\varepsilon+a-\lambda} \leqslant C .
$$

It remains to discuss the reduction of Lemma 5-5 to Lemmas 5-6 and 5-7.

From (5-2), we have

$$
N_{h}^{z} f(x)=U_{h}^{z} * f(x)+L_{h} * f(x)
$$

where

$$
\hat{U}^{z}(\xi)=\left[\rho^{*}(\xi)\right]^{z}(1-\eta(\xi)) n(\xi),
$$

and

$$
\hat{L}(\xi)=\eta(\xi) n(\xi)
$$

Now

$$
L(x)=\int_{1}^{2} \eta^{\check{ }}(x-\gamma(t)) d t .
$$

So

$$
\left\|\sup _{h>0} L_{h} * f\right\|_{p}<C_{p}\|f\|_{p}, \quad 1<p<\infty .
$$

(Again this follows by standard arguments as in Theorem 2.3 of Rivière [1971].)

Using Lemma 4-4, we have for $-a<R z<0$,

$$
U^{2}(x)=\int_{1}^{2} v^{2}(x-\gamma(t)) d t
$$

where $v^{z}$ has the following properties:

$$
\begin{aligned}
& \left|v^{z}(x)\right|<C(z) /[\rho(x)]^{a+1}, \quad x \text { large, } \\
& \left|v^{z}(x)\right|<C(z) /[\rho(x)]^{a+R z}, \quad x \text { small }
\end{aligned}
$$

$(C(z)$ as usual denotes an expression that grows at most as fast as a polynomial in $|z|)$.

Finally we choose $\psi(t)$ in $C_{0}^{\infty}(0, \infty)$ vanishing near 0 with $\psi(t)=1$ for $b_{1}<t<b_{2}$ for appropriate $b_{1}$ and $b_{2}$. Then 


$$
\begin{aligned}
U^{z}(x) \leqslant & C(z) \psi(\rho(x)) \int_{1}^{2} \frac{d t}{\{\rho(x-\gamma(t))\}^{a+R^{z}}} \\
& +C(z)(1-\psi(\rho(x))) \int_{1}^{2}\left|v^{z}(x-\gamma(t))\right| d t .
\end{aligned}
$$

The first term above can be treated by Lemmas 5-5 and 5-6, while the second term is dominated by $C(z)\{1+\rho(x)\}^{-a-1}$. Thus, we see

$$
\left\|\sup _{h>0} U_{h} * f\right\|_{p} \leqslant C(z)\|f\|_{p}, \quad 1<p \leqslant \infty .
$$

Combining (5-12), (5-11), and (5-10) we have Lemma 5-5 and the proof of part (A) of Theorem 12. by

The proof of part B is very similar to the proof of part (A). One defines $N_{h}^{z} f$

$$
\widehat{N_{h}^{2} f}(\xi)=\eta\left(\delta_{h} \xi\right) m_{h}(\xi) \hat{f}(\xi)+\left(1-\eta\left(\delta_{h} \xi\right)\right) \rho^{z}(\xi) m_{h}(\xi) \hat{f}(\xi)
$$

where

$$
m_{h}(\xi)=\frac{1}{h} \int_{h}^{2 h} \exp (2 \pi i \xi \cdot \gamma(t)) d t
$$

(See (3-6a).) Then

$$
g_{z}(f)(x)=\left\{\int_{0}^{1} \frac{d h}{h}\left|N_{h}^{z} f(x)-\phi_{h} * f(x)\right|^{2}\right\}^{1 / 2} .
$$

The estimate

$$
\left\|g_{z} f\right\|_{2} \leqslant C(z)\|f\|_{2}
$$

follows the lines of Theorem 5 part (B). The rest of the argument of Theorem 12 part (B) follows the lines of part (A).

6. A priori $L^{p}$ estimates for the maximal Hilbert transform. In this section we shall deal again with $\mathcal{F}^{*} f(x)$ and $\mathcal{F}_{1}^{*} f(x)$, defined in $\$ 3(C)$. We shall prove

THEOREM 13. (A) If $f$ is in $\delta$ and $\gamma$ is two-sided homogeneous,

$$
\left\|\mathcal{H C}^{*} f\right\|_{p} \leqslant A_{p}\|f\|_{p}, \quad 1<p<\infty .
$$

(B) Assume that for small t, $\gamma(t)$ lies in the subspace spanned by $\left\{\gamma^{(j)}(0)\right\}_{j=1}^{\infty}$. Then

$$
\left\|\mathcal{H}_{1}^{*} f\right\|_{p} \leqslant A_{p}\|f\|_{p}, \quad 1<p<\infty .
$$

We consider first part (A). Again, by an argument used in the proof of Theorem 3 we may assume that $\gamma(t)$ does not lie in a proper subspace of $R^{n}$.

We shall consider an appropriate analytic extension $\Re_{\lambda}^{2}$ of the operator $\Re_{\lambda}$ defined in $\S 3(C)$. $\Re_{\lambda}^{2} f$ will be defined for $f$ in $\delta$ by

$$
\widehat{R_{\lambda}^{z} f}(\xi)=m^{z}\left(\delta_{\lambda}^{*} \xi\right) \hat{f}(\xi)
$$

where 


$$
m^{2}(\xi)=(1-\eta(\xi))\left[\rho^{*}(\xi)\right]^{2} \int_{|t|<1} \exp \{i \xi \cdot \gamma(t)\}|t|^{2} \frac{d t}{t} .
$$

Again $\eta(\xi)$ is in $C_{0}^{\infty}$ and $\eta(\xi)=1$ near $\xi=0$.

The argument of $\S 3(C)$ gives

LEMMA 6-1. For some $\beta>0$, and $-\beta \leqslant R(z) \leqslant \beta$,

$$
\left\|\sup _{\lambda>0} \frac{1}{\lambda} \int_{0}^{\lambda} \Re_{s}^{z} f(x) d s\right\|_{2} \leqslant C(z)\|f\|_{2}
$$

for $f$ in $\mathcal{S}$. Moreover $C(z)$ may be chosen so that $|C(z)|$ grows at most as fast as a polynomial in $|z|$.

We shall show

LEMMA 6-2. For some $\beta>0$, if $0<\beta_{1}<\beta$,

$$
\left\|\sup _{\lambda>0} \Re_{\lambda}^{z} f\right\|_{p} \leqslant C(z)\|f\|_{p}, \quad 1<p<\infty,
$$

for $-\beta \leqslant R(z) \leqslant-\beta_{1} . C(z)$ as usual can be taken so that $|C(z)|$ is dominated by a polynomial in $|z|$.

Modulo technical difficulties encountered in $\$ 5$, we will then have

$$
\left\|\sup _{\lambda>0} \Re_{\lambda}^{0} f\right\|_{p} \leqslant C(p)\|f\|_{p}, \quad 1<p<\infty .
$$

These difficulties are treated as they were in $\$ 5$. (6-3) will imply Theorem 13 part (A) by the argument used in $\$ 3(\mathrm{C})$.

The derivation of Lemma 6-2 is similar in spirit to that of Lemma 5-5-namely to show that modulo trivial terms Lemma 5-6 applies. We shall content ourselves with a brief sketch of the argument.

First an application of Lemma 4-4 shows

$$
\Re_{\lambda}^{z} f(x)=L_{\lambda} * f(x)
$$

where

$$
L(x)=\int_{|t| \geq 1} l(x-\gamma(t))|t|^{2} \frac{d t}{t} ;
$$

and $l(x)$ has the following properties:

(i) $|l(x)| \leqslant C_{\alpha}(x)|x|^{-\alpha}$ for any $\alpha>0$, for large $x$ and large $\alpha$.

(ii) $l(x)$ is $C^{\infty}$ away from 0 .

(iii) $|l(x)| \leqslant C(z) /[\rho(x)]^{a+R z}$.

Thus to prove Lemma 6-2, it suffices to show

$$
\left\|\sup _{h>0} Q_{h} * f(x)\right\|_{p} \leqslant A_{p}\|f\|_{p}, \quad 1<p<\infty,
$$

where

$$
Q(x)=\int_{|t|>1} \frac{q(x-\gamma(t))}{[\rho(x-\gamma(t))]^{a-\beta}}|t|^{-\beta-1} d t ;
$$

here $q(x)$ is in $C^{\infty}$, and for any positive $\alpha$, 


$$
|q(x)| \leqslant C_{\alpha} /|x|^{\alpha} .
$$

We choose a function $\psi(t)$ in $C_{0}^{\infty}[0, \infty]$, vanishing near 0 such that $\psi(t)=1$, for $b_{1}<t \leqslant b_{2} . b_{1}$ and $b_{2}$ will depend on $\gamma$ as explained below.

We write

$$
\begin{aligned}
Q(x) & =\sum_{n=1}^{\infty} U_{n}(x)+\sum_{n=1}^{\infty} V_{n}(x), \\
& =U(x)+V(x)
\end{aligned}
$$

where

$$
U_{n}(x)=\psi\left(\frac{\rho(x)}{2^{n}}\right) \int_{2^{n}<|t|<2^{n+1}} \frac{q(x-\gamma(t))|t|^{-\beta-1}}{[\rho(x-\gamma(t))]^{a-\beta}} d t,
$$

and

$$
V_{n}(x)=\left(1-\psi\left(\frac{\rho(x)}{2^{n}}\right)\right) \int_{2^{n}<|t|<2^{n+1}} \frac{q(x-\gamma(t))}{[\rho(x-\gamma(t))]^{a-\beta}}|t|^{-\beta-1} d t .
$$

If $b_{1}$ is sufficiently small and $b_{2}$ sufficiently large,

$$
\left|V_{n}(x)\right| \leqslant \frac{C_{\alpha}}{\left[1+\rho^{\alpha}(x)\right]} \int_{2^{n}<|t|<2^{n+1}}|t|^{-\beta-1} d t
$$

for any $\alpha>0$. Thus

$$
V(x) \leqslant \sum\left|V_{n}(x)\right|<\frac{C_{\alpha}}{\left[1+\rho^{\alpha}(x)\right]},
$$

and

$$
\left\|\sup _{h>0} V_{h} * f\right\|_{p} \leqslant A_{p}\|f\|_{p}, \quad 1<p<\infty .
$$

Choose $\varepsilon>0$ such that $\alpha>\beta+\varepsilon$. Then $q(x-\gamma(t))<\rho^{-\varepsilon}(x-\gamma(t))$, so

$$
\begin{aligned}
U_{n}(x) & \leqslant \psi\left(\frac{\rho(x)}{2^{n}}\right) \int_{2^{n}<|t|<2^{n+1}}[\rho(x-\gamma(t))]^{\beta-\varepsilon-a}|t|^{-\beta-1} d t \\
& =2^{-n \varepsilon} 2^{-n a} W\left(\delta_{2^{-n} x}\right)
\end{aligned}
$$

where

$$
W(x)=\psi(\rho(x)) \int_{1<|t|<2}[\rho(x-\gamma(t))]^{\beta-\varepsilon-a}|t|^{-\beta-1} d t
$$

Now

$$
\left\|\sup _{h>0}\left|W_{h} * f\right|\right\|_{p} \leqslant A_{p}\|f\|_{p}
$$

as this is the same as the kernel treated in Lemma 5-7. So 


$$
\begin{aligned}
\left\|\sup _{h>0}\left|U_{h} * f\right|\right\|_{p} & \leqslant \sum_{n=1}^{\infty} 2^{-n \varepsilon}\left\|\sup _{h>0}\left|W_{2^{-n e}} * f\right|\right\|_{p} \\
& \leqslant \sum_{n=1}^{\infty} 2^{-n \varepsilon}\left\|\sup _{h^{\prime}>0}\left|W_{h^{\prime}} * f\right|\right\|_{p} \\
& \leqslant C A_{p}\|f\|_{p}, \quad 1<p<\infty .
\end{aligned}
$$

Now (6-5)-(6-7) imply (6-4). This completes the proof of Theorem 13 part (A). The modifications necessary for part (B) are analogous to those used in previous sections. We omit the details.

7. A priori $L^{p}$ estimates associated to spherical means. In this section we extend the results of $\$ 3$ (d) to values of $p>1$. We use the notation indicated there.

THEOREM 14. Let $f$ be in $\mathcal{S}$. The inequality

$$
\left\|\mathfrak{T}^{\alpha} f\right\|_{p} \leqslant A_{p, \alpha}\|f\|_{p}
$$

holds in the following circumstances:

(a) If $1<p<2$, when $\alpha>1-n+n / p$

(b) If $2<p<\infty$, when $\alpha>(2-n) / p$.

If $\alpha=0$, this means $n \geqslant 3, p>n /(n-1)$.

REMARK. Let

$$
f(x)= \begin{cases}|x|^{-n+1}(\log (1 /|x|))^{-1}, & |x|<\frac{1}{2}, \\ 0, & \text { otherwise. }\end{cases}
$$

Then for all sufficiently small $y$, the spherical average of $f$ on the sphere of radius $|y|$ is $\infty$. Thus $\mathscr{N}^{0}$ is unbounded in $L^{p}$ if $p<n /(n-1)$. If $n=2$, it is unknown whether $\mathfrak{T}^{0}$ is bounded in $L^{p}$ for $2<p<\infty$. Simple examples of the same kind also show that the result (a) above is sharp if $p<2$.

Theorem 14 will follow by the analytic interpolation theorem (see Stein and Weiss [1971, p. 205]). We shall use Theorem 9 as an $L^{2}$ result, and Lemma 7-1 below as $L^{p}$ and $L^{\infty}$ results:

LEMMA 7-1. $\pi^{\alpha}$ is bounded on $L^{\infty}$ if $R(\alpha)>0$ and on $L^{p}$, for every $p>1$, when $R(\alpha)>1$. Moreover if $R(\alpha)$ lies in a compact subinterval of $(0, \infty)$

$$
\log \left\|\Re^{\alpha}\right\|_{\infty}<C(\alpha)
$$

and if $R(\alpha)$ lies in a compact subset of $(1, \infty)$

$$
\log \left\|\Re^{\alpha}\right\|_{p} \leq C(\alpha) /(p-1),
$$

where $C(\alpha)$ grows at most as fast as a polynomial in $|\alpha|$.

Lemma $7-1$ is a consequence of the standard maximal theorem and the known fact: for $R(\alpha)>0$,

$$
m^{\alpha}(\xi)=\hat{N}_{\alpha}(\xi)
$$

where 


$$
\begin{gathered}
N_{\alpha}(x)= \begin{cases}\Delta(n, \alpha)\left(1-|x|^{2}\right)^{\alpha-1}, & |x| \leqslant 1, \\
0, & |x| \geqslant 1,\end{cases} \\
\Delta(n, \alpha)=\frac{\Gamma\left(\frac{n}{2}+\alpha\right)}{\pi^{n / 2} \Gamma(\alpha)} .
\end{gathered}
$$

(See e.g. Stein and Weiss [1971, p. 171].)

The technical details of the interpolation argument are the same as those of 85.

8. Extensions from a priori inequalities. Our goal here will be to extend previous results, which have been limited to the class $\mathcal{S}$, to arbitrary $L^{p}$ functions.

(A) Maximal functions and maximal Hilbert transforms associated to curves. We begin with the following simple lemma:

LEMMA 8-1. Let $f(x)$ be a locally integrable function, and let $\gamma(t)$ be a continuous curve. Then $f(x-\gamma(t))$ is a measurable function of $x$ and $t$. Also for almost every $x, f(x-\gamma(t))$ is a measurable and locally integrable function of $t$.

We shall first show that $f(x-\gamma(t))$ is jointly measurable in $x$ and $t$. To do this we need to show that if $E$ is a measurable set in $R^{n+1}$, then the set $\{(x, t) \mid x-\gamma(t) \in E\}$ is again a measurable set in $R^{n+1}$. If $E$ were Borel measurable, this would be obvious. Also if $E$ is of measure zero, then for each $t$, the set of $x$ 's such that $x-\gamma(t) \in E$ is of measure zero. Thus by Fubini's Theorem, if $E$ is of measure zero, then the set $\{x, t \mid x-\gamma(t) \in E\}$ has measure zero. Since an arbitrary Lebesgue measurable set is the union of a Borel set and a set of Lebesgue measure zero, the measurability assertion of the lemma is proved. The fact that $f(x-\gamma(t))$ is locally integrable in $t$ for almost every $x$ follows from Fubini's Theorem.

Lemma 8-1 implies the following two facts for locally integrable $f$ :

$$
M_{h} f(x)=\frac{1}{h} \int_{0}^{h} f(x-\gamma(t)) d t
$$

is a well-defined measurable function of $x$ for every positive $h$, and $M_{h} f(x)$ is a continuous function of $h$ for almost every $x$. Also

$$
H_{\varepsilon, N} f(x)=\int_{\varepsilon<|t|<N} f(x-\gamma(t)) \frac{d t}{t}
$$

is a well-defined measurable function of $x$ for each fixed positive $\varepsilon$ and $N$. Moreover for almost every $x, H_{\varepsilon, N} f(x)$ is continuous in $\varepsilon$ and $N$. From (8-2), we have

$$
\Re f(x)=\sup _{h>0}\left|M_{h} f(x)\right|=\sup _{\substack{r>0 \\ \text { rational }}}\left|M_{r} f(x)\right|
$$

for almost every $x$, and

$$
\mathcal{C} * f(x)=\sup _{\substack{\varepsilon>0 \\ N>0}}\left|H_{\varepsilon, N} f(x)\right|=\sup _{\substack{r_{1}>0 \\ r_{2}>0 \\ r_{1}, r_{2} \text { rational }}}\left|H_{r_{1}, r_{2}} f(x)\right|
$$


for almost every $x$.

This implies that $\mathscr{N} f$ and $\mathcal{H}^{*} f$ are measurable. Furthermore, one can see that there exist positive, rational-valued measurable functions $h(x), \varepsilon(x)$, and $N(x)$ such that

$$
\left|M_{h(x)} f(x)\right|>\frac{1}{2} \Re f(x) \text { a.e., }
$$

and

$$
\left|H_{\varepsilon(x), N(x)} f(x)\right|>\frac{1}{2} \mathcal{H} * f(x) \text { a.e. }
$$

Now by an easy limiting argument from Theorems 12 and 13 one obtains

THEOREM 8. (A) For any $f$ in $L^{p}, 1<p \leqslant \infty$, and $\gamma(t)$ homogeneous, $N f$ is in $L^{p}$, and

$$
\|\mathfrak{T} f\|_{p} \leqslant A_{p}\|f\|_{p}
$$

For any $f$ in $L^{p}, 1<p<\infty$, and $\gamma(t)$ two-sided homogeneous,

$$
\|\mathcal{F C} * f\|_{p} \leqslant A_{p}\|f\|_{p} \text {. }
$$

(B) Let $\gamma(t)$ be $C^{\infty}$. Assume that for small $t, \gamma$ lies in the subspace spanned by $\gamma^{(j)}(0), j=1,2, \ldots$ Then if $f$ is in $L^{p}, 1<p \leqslant \infty$, $\mathfrak{N}_{1} f$ is in $L^{p}$, and

$$
\left\|\Re_{1} f\right\|_{p} \leqslant A_{p}\|f\|_{p} \text {. }
$$

If $f$ is in $L^{p}, 1<p<\infty, \mathcal{H}_{1}^{*}$ f is in $L^{p}$ and

$$
\left\|\mathcal{F}_{1}^{*} f\right\|_{p} \leqslant A_{p}\|f\|_{p} .
$$

(We have not actually discussed part (B), but the discussion is the same as that of part (A).)

Standard arguments then give the following corollaries:

COROLlaRY 8-6. Let $\gamma(t)$ satisfy the hypotheses of (A) or (B) of Theorem 14, then if $f$ is locally in $L^{p}, p>1$,

$$
\lim _{h \rightarrow 0} \frac{1}{h} \int_{0}^{h} f(x-\gamma(t)) d t=f(x) \text { a.e. }
$$

Corollary 8-7. (A) If $\gamma(t)$ is two-sided homogeneous, and $f$ is in $L^{p}$, $1<p<\infty$,

$$
\mathcal{H} f(x)=\lim _{\substack{\varepsilon \rightarrow 0 \\ N \rightarrow \infty}} \mathcal{H}_{\varepsilon, N} f(x)
$$

exists almost everywhere and in $L^{p}$. Moreover,

$$
\|\mathcal{H} f\|_{p} \leqslant A_{p}\|f\|_{p}, \quad 1<p<\infty .
$$

(B) Let $\gamma(t)$ be in $C^{\infty}$. Assume that for small $t, \gamma$ lies in the subspace spanned by $\gamma^{(j)}(0), j=1,2, \ldots$ Then

$$
\mathcal{H}_{1} f(x)=\lim _{\varepsilon \rightarrow 0} \mathcal{H}_{\varepsilon, 1} f(x)
$$

exists almost everywhere and in $L^{p}$. Moreover

$$
\left\|\mathcal{G}_{1} f\right\|_{p} \leqslant A_{p}\|f\|_{p}, \quad 1<p<\infty .
$$

(B) Maximal spherical averages. Here we shall restrict our attention to 
dimension $n \geqslant 3$. We set $S_{x}(t)=\left\{y\right.$ in $\left.R^{n}|| x-y \mid=t\right\}$, and let $\sigma_{t, x}$ denote the usual rotationally invariant measure on $S_{x}(t)$. The following lemma will be critical for us (and interesting in its own right).

LEMMA 8-8.9 Let $E$ be a set of measure zero in $R^{n}, n \geqslant 3$. Then for almost every $x$ in $R^{n}$

$$
\sigma_{t, x}\left(E \cap S_{x}(t)\right)=0
$$

for every $t>0$.

Before proving Lemma 8-8, we need to make some preliminary remarks.

In $\$ 3(d)$, we defined

$$
M_{\downarrow} f(x)=\int_{|y|=1} f(x-t y) d \sigma(y)=\int_{|y|=1} f(x-t y) d \sigma_{1,0}(y) .
$$

Since the intersection of a Borel set in $R^{n}$ with an $n$-1-dimensional sphere is Borel measurable (with respect to the Borel $\sigma$-algebra on the sphere), we see that $M_{t} f(x)$ is well defined for any Borel measurable function $f$ and every $x$ and $t$. Thus if $f$ is nonnegative and Borel measurable

$$
\mathfrak{N} f(x)=\sup _{t>0}\left|M_{t} f(x)\right|
$$

is well defined (possibly $+\infty$ ) for every $x$.

We shall need the following

LEMMA 8-9. Let $f(x)$ and $f_{n}(x), n=1,2, \ldots$, be nonnegative Borel measurable functions. Assume $f_{n}(x)$ increases to $f(x)$ for every $x$, then

$$
\Re f(x)=\lim _{n \rightarrow \infty} \Re f_{n}(x) .
$$

Proof. Clearly

$$
\Re f(x) \geqslant \Re f_{n}(x) \text { for every } n
$$

Next

$$
M_{\imath} f_{n}(x) \rightarrow M_{\imath} f(x)
$$

for every $t$ and $x$ by the monotone convergence theorem. Thus

$$
\lim _{n \rightarrow \infty} \Re f_{n}(x) \geqslant M_{\downarrow} f(x)
$$

for every $t$. So

$$
\varliminf_{n \rightarrow \infty} \mathfrak{N} f_{n}(x) \geqslant \mathscr{N} f(x) .
$$

(8-10) and (8-11) imply Lemma 8-9.

From Lemma 8-9, we see that if $f$ is the characteristic function of an open set, $\Re f(x)$ is measurable. Moreover, using Theorem 14 (with $\alpha=0$ ) we have that

$$
\|\Re f\|_{p}<C_{p}\|f\|_{p}, \quad p>n /(n-1) .
$$

Now it is easy to prove Lemma 8-8. We let $E_{1}$ be a Borel set of measure

\footnotetext{
${ }^{9}$ Here we follow a suggestion of A. Calderon and A. Zygmund.
} 
zero containing $E$, and let $g$ be the characteristic function of $E_{1}$. It suffices to show $\Re g(x)$ is zero almost everywhere. Now let $\theta_{l}$ be a sequence of open sets containing $E_{1}$ such that the measure of $\theta_{l}$ tends to 0 . Let $f_{l}$ be the characteristic function of $\mathcal{O}_{l}$. Then

$$
\Re g(x) \leqslant \underset{l \rightarrow \infty}{\lim } \Re f_{l} .
$$

But

$$
\left\|\lim _{l \rightarrow \infty} \Re f_{l}\right\|_{2} \leqslant \underset{l \rightarrow \infty}{\lim }\left\|\Re f_{l}\right\|_{2}=0
$$

by (8-12). Thus $\lim _{l \rightarrow \infty} \mathfrak{T} f_{l}=0$ almost everywhere, so $\Re g(x)=0$ almost everywhere. This concludes the proof of Lemma 8-8.

Lemma 8-8 has the following consequence:

(8-13) If $E$ is Lebesgue measurable then for almost every $x$ and every $t$, $S_{x}(t) \cap E$ is Lebesgue measurable (with respect to Lebesgue measure on $\left.S_{x}(t)\right)$.

This implies that if $f(x)$ is a simple Lebesgue measurable function, for almsot every $x, f$ restricted to $S_{x}(t)$ is measurable for all $t$. Since any Lebesgue measurable function is everywhere the limit of a sequence of simple functions, we see that for almost every $x, f$ restricted to the sphere $S_{x}(t)$ is measurable for every $t$. Thus if $f \geqslant 0$ and is Lebesgue measurable then for almost every $x, M_{t} f(x)$ is defined for all $t$.

Hence

$$
\mathscr{T} f(x)=\sup _{t>0} M_{t} f(x)
$$

is defined for almost every $x$. We now prove

THEOREM 16. Let $f \geq 0$, then $\Re f(x)$ is measurable. If $f$ is in $L^{p}$ for $p>n /(n-1)$, so is $\Re$ f and

$$
\|\Re f\|_{p}<A_{p}\|f\|_{p} .
$$

Proor. The result is first established for decreasing limits of characteristic functions of open sets from (8-12). Lemma 8-8 then implies the theorem for characteristic functions of measurable sets. Thus it is also true for simple functions. Finally we conclude Theorem 16 in general since we can now assert the conclusion of Lemma 8-9 under the more general conditions that $f_{n}$ and $f$ are Lebesgue measurable.

Theorem 16 leads to the following corollary

COROLLARY 8-14. If $f$ is locally in $L^{p}, p>n /(n-1), n \geq 3$, then

$$
M_{h} f(x) \rightarrow f(x) \text { a.e. }
$$

Remark. It has been pointed out to us by R. Coifman and G. Weiss and independently by $\mathrm{C}$. Calderón that by using ideas similar to the proof of Theorem 14 one can show that

$$
\lim _{k \rightarrow \infty} M_{2^{-k}}(f)(x)=f(x) \text { a.e. }
$$

(as $k$ ranges over integers) whenever $f \in L^{p}, 1<p$, and for any $n \geqslant 2$. 


\section{PART III-EPILOGUE}

1. Some areas not covered. We want to make some brief comments about several additional topics related to our main subject.

(i) As we indicated at the beginning of this paper the problem of considering other sets besides balls (or cubes) in differentiation theory in $R^{n}$ has been attracting increasing attention lately. We should mention in this connection the interesting work of Cordoba and R. Fefferman [1975], [1977] and Strömberg [1976] dealing with rectangles in differentiation theory. Several earlier results and some background may be found in the survey of de Guzman [1975].

(ii) The exploitation of curvature of certain sets via the Fourier transform has its roots in the problem of estimation of the number of lattice points in such sets. These techniques were, in effect, introduced by van der Corput more than fifty years ago. Generalizations and more recent results may be found in the papers of Hlawka [1950], Herz [1962b] and Randol [1966].

A topic having some tangential relation with this and also with the spherical maximal functions discussed in detail above is the problem of restricting the Fourier transform to lower-dimensional varieties. A particular question which is of some interest is whether there is an a-priori inequality of the form

$$
\left(\int_{|\xi|=1}|\hat{f}(\xi)|^{q} d \sigma(\xi)\right)^{1 / q} \leqslant A_{p, q}\left(\int_{R^{n}}|f(x)|^{p} d x\right)^{1 / p}
$$

for suitable $p$ and $q$.

About ten years ago it was observed that indeed such inequalities held, when $n \geqslant 2$, as long as $p$ is sufficiently close to 1 . More recently the best possible results were proved for $n=2$, i.e. $q=p^{\prime} / 3,1 \leqslant p<4 / 3$, and for general $n$ when $q=2$, and $1 \leqslant p \leqslant(2 n+2) /(n+3)$. (Further details may be found in the papers of C. Fefferman [1970], Zygmund [1974], and Tomas [1975]; however the sharp result for $q=2$ is still unpublished.) In view of the above discussion it may be appropriate to record here the following problem.

Problem 1. Prove that (1.1) holds when $n \geqslant 3, q=((n-1) /(n+1)) p^{\prime}$, and $1 \leqslant p<2 n /(n+1)$.

2. Some further problems. We state here several problems which arise naturally from our work, but whose solution would seem to require new ideas. The first four need little comment.

Problem 2. (a) Is the spherical maximal function bounded in $L^{p}$ for any $p<\infty$ in two dimensions?

(b) Does the corresponding differentiation theorem hold at least for bounded functions?

Problem 3. Is there an $L^{1}$ theory for $\Re$ and $\mathscr{H}$ corresponding to the curves $\gamma$ considered in this paper? In particular, does the differentiation theorem hold for all locally integrable $f$ ?

Problem 4. Is there a covering lemma (analogous to 14 (b) in Part I) which will prove results about $\mathfrak{N}$ ?

This problem might have applications to the next one. 
Problem 5. What can be said if $\gamma(t)=\gamma(t, x)$ can vary with $x$ ? For example, is it true that

$$
\lim _{\varepsilon \rightarrow 0} \frac{1}{\varepsilon} \int_{0}^{\varepsilon} f\left(x_{1}-t, x_{2}-a\left(x_{1}, x_{2}\right) t^{2}\right) d t=f\left(x_{1}, x_{2}\right) \text { a.e. }
$$

for every bounded $f$ if (say) $a$ is smooth and positive?

The following problem arises from the observation that the (standard) $g$-function

$$
\left[\int_{0}^{\infty} y\left|\frac{\partial u(x, y)}{\partial y}\right|^{2} d y\right]^{1 / 2},
$$

(where $u$ is the Poisson integral of $f$ ) is pointwise majorized by the $g$-function

$$
\left[\int_{0}^{\infty} t\left|\frac{d M_{t}(f)}{d t}\right|^{2} d t\right]^{1 / 2}
$$

used in $\$ 4$ of Part I.

Problem 6. (a) Explore the relationship between the g-functions used for maximal spherical averages and the standard $g$-functions.

(b) Clarify the connection between the $g$-functions associated to homogeneous curves and the more standard ones arising for nonisotropic dilations as in Calderón and Torchinsky [1975].

The next problem requires several definitions. Let $S=G / K$ be a symmetric space (of noncompact type). On it we can define a natural maximal function $M$ by

$$
M(f)(x)=\sup _{r>0}\left(\frac{1}{m(B(x, r))} \int_{B(x, r)}|f(y)| d m(y)\right)
$$

where $B(x, r)$ is the geodesic ball centered at $x$ of radius $r$-the geodesics being taken with respect to a $G$-invariant Riemannian metric on $S ; m$ is the induced invariant measure on $S$.

The obstacle to proving $L^{p}$ and $L^{1}$ estimates for $M$ by the usual methods is the exponential growth of $m(B(x, r))$, as $r \rightarrow \infty$, which reflects the (negative) curvature of $S$. Nevertheless, implicitly using the Fourier analysis on $S$ (more precisely, estimates for spherical functions) Clerc and Stein [1974] proved that $\|M(f)\|_{p} \leqslant A_{p}\|f\|_{p}, 1<p \leqslant \infty$. Those methods however do not dispose of the case $p=1$.

Problem 7. (a) Is there an $L^{1}$ theory for the maximal function of symmetric spaces of noncompact type?

(b) Is there a covering lemma which gives the results for this maximal function?

Another problem of a different type is whether the results for $\mathcal{H}$ and $\Re$ for homogeneous curves are extendable to multiparameter sets. We shall, for the sake of simplicity, keep our discussion to 3 dimensions and 2 parameters.

Problem 8. Are there positive results for

$$
\mathcal{H C} f(x, y, z)=\iint f(x-s, y-t, z-\gamma(s, t)) \frac{d s d t}{s t},
$$


and

$$
\Re f(x, y, z)=\sup _{h, k>0} \frac{1}{h k} \int_{0}^{h} \int_{0}^{k} f(x-s, y-t, z-\gamma(s, t)) d s d t ?
$$

For $\mathcal{K}$ a positive result is known for $p=2$ if $\gamma(s, t)=|s|^{\alpha}|t|^{\beta}$. (See Nagel and Wainger [1977].) There it is also shown that $\mathcal{H C}$ "generates" singular integral operators where the kernels are not integrable across positive dimensional varieties.

3. Some further results and counterexamples. The last problem we wish to discuss can be stated as follows

Problem 9. Fix $p, 1<p<\infty$. Determine the class of curves $\gamma$ for which there are $L^{p}$ results for $\mathcal{H}$ and $\mathscr{N}$ corresponding to $\gamma$.

The nature of this problem will be clarified by several examples presented below. The simplest is as follows.

Let $\gamma(t)$ be an odd function such that $\gamma(t)=t$ for $0 \leqslant t \leqslant 1$, and $\gamma(t)=a t$ $+b$ for $t>1$, with $b \neq 0$. We shall show that the Hilbert transform corresponding to $(t, \gamma(t))$ is unbounded in $L^{2}$. Thus in effect we wish to see that

$$
\int_{0}^{N} \sin (\xi t+\eta \gamma(t)) \frac{d t}{t}
$$

is unbounded. Now $\int_{0}^{1} \sin (\xi t+\eta \gamma(t)) d t / t$ is bounded; but

$$
\begin{aligned}
\int_{1}^{N} \sin (\xi t & +\eta \gamma(t)) \frac{d t}{t} \\
= & \cos b \eta \int_{1}^{N} \sin (\xi t+a \eta t) \frac{d t}{t} \\
& +\sin b \eta \int_{1}^{N} \cos (\xi t+a \eta t) \frac{d t}{t}
\end{aligned}
$$

The first integral is clearly bounded, and the second is unbounded.

The main idea here is, of course, to construct a function $\gamma(t)$ such that $(t, \gamma(t))$ is flat and such that $\sin (\xi t+\eta \gamma(t))$ is not zero for a sufficiently large set of $t$ 's.

Next by taking $\gamma(t)$ so that $(t, \gamma(t))$ has many "long" straight line segments not passing through the origin, one can construct $C^{\infty}$ curves such that

$$
f \rightarrow \mathscr{K}_{1} f=\int_{-1}^{1} f(x-t, y-\gamma(t)) \frac{d t}{t}
$$

is unbounded in $L^{2}$. Modifying this idea one can find odd functions $\gamma(t)$ such that $\gamma^{\prime \prime}(t) \rightarrow \infty$ as $t \rightarrow \infty$, while

is unbounded in $L^{2}$.

$$
f \rightarrow \int_{|t|>1} f(x-t, y-\gamma(t)) \frac{d t}{t}
$$

On the other hand if $\gamma(t)$ is odd, $\gamma(0)=0, \gamma$ is $C^{2}$, and $\gamma^{\prime \prime}(t)$ tends to infinity monotonically as $t$ tends to infinity, then

$$
f \rightarrow \int_{-\infty}^{\infty} f(x-t, y-\gamma(t)) \frac{d t}{t}
$$


is bounded in $L^{p}\left(R^{2}\right)$, at least for $5 / 3<p<5 / 2$. We shall not give any. details here, but refer the reader to Nagel and Wainger [1976] where also other more technical results are discussed.

We shall now discuss corresponding results for maximal functions associated to curves. The first curve we consider is the plane curve $(t, \gamma(t))$, where $\gamma(t)$ behaves like $\log t$ for large $t$. If $f$ is the characteristic function of the infinite strip $-\ln 2<y<0$. Then for some $y_{0}$ and $y>y_{0}$

$$
\frac{1}{h} \int_{0}^{h} f(x-t, y-\gamma(t)) d t=\frac{1}{2}
$$

if $h=\ln 2 y$. If one lets $f$ be the characteristic function of $-\ln 2<y<0$, $-N<x<N$, the same reasoning shows $|\Re f|>1 / 4$ on a set of measure $C N \log N$. Thus for this curve

$$
\|\Re f\|_{p} \leqslant A_{p}\|f\|_{p}
$$

holds only for $p=\infty$.

Our next goal will be to exhibit a $C^{\infty}$ curve $\bar{\gamma}(t)$ such that

$$
\Re_{1} f(x)=\sup _{1>h>0}\left|\frac{1}{h} \int_{0}^{h} f(x-\bar{\gamma}(t)) d t\right|
$$

is unbounded on each $L^{p}, p<\infty . \bar{\gamma}(t)$ will be a plane curve $\left(t, \gamma_{1}(t)\right)$. We begin by considering a preliminary curve $(t, \gamma(t))$, where

$$
\gamma(t)=\left\{2^{-2^{n}} \mid 2^{-n}<t<2 \cdot 2^{-n}, n=1,2, \ldots\right\} .
$$

Let $f(x, y)$ be the characteristic function of the rectangle $-1<x<1$, $0<y<\varepsilon$. It is easy to see that $\Re_{1} f>1 / 4$ in the rectangle $2^{-n}<x<2^{-n}$ $+1,2^{-2^{n}}<y<2^{-2^{n}}+\varepsilon$. Moreover, one easily sees that $C \log \log 1 / \varepsilon$ of these rectangles are disjoint. Thus $\mathfrak{T}_{1} f>1 / 4$ on a set of measure $C \varepsilon \log$ $\log 1 / \varepsilon$, and hence

$$
\left\|\Re_{1} f\right\|_{p} \leqslant A(p)\|f\|_{p}
$$

can hold only for $p=\infty$. It is easy to modify the above example to make $\gamma$ $C^{\infty}$ since the jumps of $\gamma$ are exponentially smaller than the lengths of the intervals of constancy.

One may also deduce from the estimates above the fact that the differentiation theorem does not hold for $\bar{\gamma}(t)$, even for the class of bounded functions.

We shall formulate the required argument as a lemma, in the setting of the $n$-torus instead of $R^{n}$. Thus we shall think of our functions as defined on the fundamental cube $\left\{x \mid-1 / 2<x_{j}<1 / 2\right\}$ in $R^{n}$, and continued periodically. We let $\left\{T_{k}\right\}$ be a sequence of operators given by $T_{k} f=f * d \mu_{k}$, where each $d \mu_{k}$ is a nonnegative measure whose total mass is bounded (in $k$ ). We assume that

$$
\lim _{k \rightarrow 0} T_{k}(f)=f \quad \text { a.e. for every } f \in L^{\infty} .
$$

We set $T^{*}(f)=\sup _{k}\left|T_{k}(f)\right|$.

LemMa. Assume that $\left\{T_{k}\right\}$ satisfies the above assumption, including (2.1). Then for every $c>0$ there exists $A=A_{c}$ so that 


$$
\left|\left\{x \mid T^{*}\left(\chi_{E}\right)>c\right\}\right| \leqslant A_{c}|E|,
$$

for all measurable sets $E$.

Note that by the previous construcion (2.3) fails (when $c=1 / 4$ ), and so differentiation almost everywhere fails for the curve $\bar{\gamma}$ for bounded functions.

Proof of THE LemMa. Suppose (2.3) does not hold for some $c$. Then there exists a sequence of sets $E_{n}$, so that if $E_{n}^{*}=\left\{x \mid T^{*} \chi_{E_{n}}>c\right\}$, then $\left|E_{n}^{*}\right| /\left|E_{n}\right| \rightarrow$ $\infty$, as $n \rightarrow \infty$. Now set $T_{r}^{*}(f)=\sup _{n \geqslant r}\left|T_{n} f\right|$.

By using the sets $E_{n}$, together with repetitions and a selected subsequence, it is not difficult to construct another collection of sets $F_{n}$, with the following properties. There exists an integer-valued function $n \rightarrow r(n)$, with $r(n) \rightarrow \infty$, as $n \rightarrow \infty$, so that if $F_{n}^{*}=\left\{x \mid T_{r(n)}^{*}\left(\chi_{F_{n}}\right)>c\right\}$, then

$$
\sum\left|F_{n}\right| \leqslant 1 / 2 \text {, while } \Sigma\left|F_{n}^{*}\right|=\infty \text {. }
$$

By Calderón's lemma (see Zygmund [1959, II, 165]), there is a sequence $\left\{x_{n}\right\}$ of points, so the translated sets $F_{n}^{*}+x_{n}$ have the property that almost every point is contained in infinitely many of the sets $F_{n}^{*}+x_{n}$.

Let $F=\bigcup\left\{F_{n}+x_{n}\right\}$. Then $|F| \leqslant 1 / 2$, but $\lim \sup _{n \rightarrow \infty} T_{n}\left(\chi_{F}\right) \geqslant c$ almost everywhere. This is a contradiction, and the lemma is proved. We conclude with a result somewhat paradoxical in view of the above counterexample-that the differentiation theorem may hold even in some "infinitely flat" cases-besides the straight line. We consider the curve $(t, \gamma(t))$ in $R^{2}$, and outline the proof of the following theorem for $p=2$.

THEOREM. If $\gamma(0)=\gamma^{\prime}(0)=0$, and $\gamma(t), \gamma^{\prime}(t)$, and $\gamma^{\prime \prime}(t)$ are increasing, and positive when $t>0$, then

$$
\|\Re f\|_{2} \leqslant A\|f\|_{2} \text {. }
$$

This theorem is proved by introducing a suitable $g$-function. We set

$$
M_{h} f(x, y)=\frac{1}{h} \int_{h}^{2 h} f(x-t, y-\gamma(t)) d t,
$$

and

$$
N_{h} f(x, y)=\frac{1}{h \gamma(h)} \int_{h}^{2 h}\left\{\int_{0}^{\gamma(h)} f(x-t, y-s) d s\right\} d t .
$$

Then define

$$
g(f)(x, y)=\left\{\int_{0}^{\infty} \frac{d h}{h}\left|M_{h} f(x, y)-N_{h} f(x, y)\right|^{2}\right\}^{1 / 2} .
$$

By the reasoning of $\S(3-6)$, it suffices to prove $\|g(f)\|_{2} \leqslant c\|f\|_{2}$. Following the lines of (3-6), this estimate in turn follows from

$$
\int_{0}^{\infty} \frac{d h}{h}\left|m_{h}(\xi, \eta)-n_{h}(\xi, \eta)\right|^{2} \leqslant c<\infty,
$$

where

$$
m_{h}(\xi, \eta)=\frac{1}{h} \int_{h}^{2 h} \exp \{i \xi t+i \eta \gamma(t)\} d t
$$


and

$$
n_{h}(\xi, \eta)=\frac{1}{h \gamma(h)} \int_{h}^{2 h}\left\{\int_{0}^{\gamma(h)} \exp \{i \xi t+i \eta(s)\} d s\right\} d t
$$

We divide the integral in (1) into three parts: $0 \leqslant h \leqslant \frac{1}{2} \gamma^{-1}(1 / \eta)$, $\frac{1}{2} \gamma^{-1}(1 / \eta)<h<\gamma^{-1}(1 / \eta)$, and $h>\gamma^{-1}(1 / \eta)$. The boundedness of the integral over the first interval follows from the estimate $\left|m_{h}(\xi, \eta)-n_{h}(\xi, \eta)\right|$ $<\eta \gamma(2 h)$. The boundedness over the second part is obvious. The boundedness over the third part is proved by noting that

$$
\left|m_{h}(\xi, \eta)\right| \leqslant c / h\left\{\eta \gamma^{\prime \prime}(h)\right\}^{1 / 2}
$$

(by van der Corput's lemma) and

$$
\left|n_{h}(\xi, \eta)\right| \leqslant 1 / \eta \gamma(h) \text {. }
$$

Throughout one needs the estimates $\gamma(t) \leqslant t \gamma^{\prime}(t)$ and $\gamma^{\prime}(t) \leqslant t \gamma^{\prime \prime}(t)$.

ADDED IN PROOF. Recently Fourier transform methods have been used to improve the results of J. Strömberg [1976] and A. Cordoba and R. Fefferman [1977]. See Differentiation in lacunary directions, by A. Nagel, E. M. Stein and S. Wainger, Proc. Nat. Acad. Sci. U.S.A. 75 (1978), 1060-1062.

Also A. Nagel, E. M. Stein and S. Wainger have recently made progress in problem 5) above. A summary of these results will appear shortly.

\section{REFERENCES}

D. Burkholder and R. Gundy [1970], Extrapolation and interpolation of quasi-linear operators on martingales, Acta Math. 124, 249-304.

H. Buseman and W. Feller [1934], Differentiation der L-integrale, Fund. Math. 22, 226-256.

A. P. Calderón and A. Torchinsky [1975], Parabolic maximal functions associated with a distribution, Advances in Math. 16, 1-64.

A. P. Calderón and A. Zygmund [1952], On the existence of certain singular integrals, Acta Math. 88, 85-139.

[1956], On singular integrals, Amer. J. Math. 18, 289-309.

J. L. Clerc and E. M. Stein [1974], $L^{P}$ multipliers for non-compact symmetric spaces, Proc. Nat. Acad. Sci. U.S.A. 71, 3911-3912.

R. Coifman [1972], Distribution function inequalities for singular integrals, Proc. Nat. Acad. Sci. U.S.A. 69, 2838-2839.

A. Cordoba and R. Fefferman [1975], A geometric proof of the strong maximal theorem, Ann. of Math. (2) 102, 95-100.

[1977], On differentiation of integrals, Proc. Nat. Acad. Sci. U.S.A. 74, 2211-2213.

A. Erdélyi [1953], Higher transcendental functions, vol. 2, McGraw-Hill, New York.

E. B. Fabes [1966], Singular integrals and partial differential equations of parabolic type, Studia Math. 28 81-131.

E. B. Fabes and N. M. Rivière [1966], Singular integrals with mixed homogeneity, Studia Math. 27, 19-38.

[1967], Symbolic calculus of kernels with mixed homogeneity, Proc. Sympos. Pure Math., vol. 10, Amer. Math. Soc., Providence, R.I.

C. Fefferman [1970], Inequalities for strongly singular convolution operators, Acta Math. 124, 9-36.

C. Fefferman and E. M. Stein [1972], $H^{p}$ spaces of several variables, Acta Math. 229, 137-193.

G. B. Folland and E. M. Stein [1974], Estimates for the $\bar{\partial}_{b}$ complex and analysis on the Heisenberg group, Comm. Pure Appl. Math. 27, 429-522.

M. de Guzman [1975], Differentiation of integrals in $R^{n}$, Springer-Verlag, Berlin and New York.

E. Hlawka [1950], Uber Integrale auf konvexen Körpern. I, Monatsh. Math. 54, 1-36. 
G. H. Hardy [1949], Divergent series, Clarendon Press, Oxford.

G. H. Hardy and J. E. Littlewood [1930], A maximal theorem with function-theoretic applications, Acta Math. 54, 81-116.

C. S. Herz [1962a], Fourier transforms related to convex sets, Ann. of Math. (2) 75, 81-92. , [1962b], On the number of lattice points in a convex set, Amer. J. Math. 84, 126-133.

L. Hörmander [1967], Hypoelliptic second order differential equations, Acta Math. 119, 147-171.

B. F. Jones [1964], A class of singular integrals, Amer. J. Math. 86, 441-462.

S. Kaczmarz and H. Steinhaus [1951], Theorie der Orthogonalreihen, Chelsea, New York.

A. W. Knapp and E. M. Stein [1971], Intertwining operators for semi-simple Lie groups, Ann. of Math. (2) 93, 489-578.

A. Koranyi [1972], Harmonic functions on symmetric spaces, Symmetric Spaces, Boothby and Weiss, eds., Marcel Dekker, New York, pp. 379-412.

J. E. Littlewood and R.E.A.C. Paley [1931], [1936], [1937], Theorems on Fourier Series and Power Series, (I), J. London Math. Soc. 6, 230-233; (II) Proc. London Math. Soc. 42, 52-89; (III) ibid. 43, 105-126.

W. Littman [1963], Fourier transforms of surface-carried measures and differentiability of surface averages, Bull. Amer. Math. Soc. 69, 766-770.

J. Marcinkiewicz and A. Zygmund [1939], On the summability of double Fourier series, Fund. Math. 32, 112-132.

S. Mihlin [1950], Singular integral equations, Amer. Math. Soc. Transl. (1) 10 (1962), 84-198.

A. Nagel, N. M. Rivière, and S. Wainger [1974], On Hilbert transforms along curves, Bull. Amer. Math. Soc. 80, 106-108. , [1976a], On Hilbert transforms along curves, II, Amer. J. Math. 98, 395-403.

$73,1416-1417$.

[1976b], A maximal function associated to the curve $\left(t, t^{2}\right)$, Proc. Nat. Acad. Sci. U.S.A.

A. Nagel and S. Wainger [1976], Hilbert transforms associated with plane curves, Trans. Amer. Math. Soc. 223, 235-252.

[1977], $L^{2}$ boundedness of Hilbert transforms along surfaces and convolution operators homogeneous with respect to a multiple parameter group, Amer. J. Math. 99, 761-785.

B. Randol [1966], A lattice-point problem, Trans. Amer. Math. Soc. 121, 257-268.

[1972], The asymptotic behavior of a Fourier transform and the localization property for

eigenfunction expansions, Trans. Amer. Math. Soc. 168, 265-271.

N. M. Rivière [1971], Singular integrals and multiplier operators, Ark. Mat. 9, 243-278.

L. Rothschild and E. Stein [1976], Hypoelliptic differential operators and nilpotent groups, Acta Math. 137, 247-320.

E. M. Stein [1961], On limits of sequences of operators, Ann. of Math. (2) 74, 140-170. [1970a], Singular integrals and differentiability properties of functions, Princeton Univ. Press, Princeton, N.J.

[1970b], Topics in harmonic analysis related to the Littlewood-Paley theory, Princeton

Univ. Press, Princeton, N. J.

[1971], Some problems in harmonic analysis suggested by symmetric spaces and semi-

simple Lie groups, Actes, Internat. Congr. Math., 1, pp. 173-189.

$21 \overline{74-2175}$.

[1976a], Maximal functions: Spherical means, Proc. Nat. Acad. Sci. U.S.A. 73,

[1976b], Maximal functions: homogeneous curves, Proc. Nat. Acad. Sci. U.S.A. 73, 2176-2177.

[1976c], Maximal functions: Poisson integrals on symmetric spaces, Proc. Nat. Acad. Sci.

U.S.A. 73, 2547-2549.

E. M. Stein and S. Wainger [1970], The estimation of an integral arising in multiplier transformations, Studia Math. 35, 101-104.

pp. $4295-4296$.

[1976], Maximal functions associated to smooth curves, Proc. Nat. Acad. Sci. U.S.A. 73,

E. M. Stein and G. Weiss [1971], Introduction to Fourier analysis on Euclidean spaces, Princeton Univ. Press, Princeton, N.J.

J. O. Strömberg [1976], (a) Weak estimates for maximal functions with rectangles in certain directions, Institut Mittag-Leffler, report \# 10, Ark. Mat. 15 (1977), 229-240.

E. C. Titchmarsh [1937], Introduction to the theory of the Fourier integral, Oxford Univ. Press, London. 
P. Tomas [1975], A restriction theorem for the Fourier transform, Bull. Amer. Math. Soc. 81, 477-478.

F. Treves [1975], Basic linear partial differential equations, Academic Press, New York.

N. Wiener [1939], The ergodic theorem, Duke Math. J. 5, 1-18.

K. Yosida [1965], Functional analysis, Springer-Verlag, Berlin and New York.

F. Zo [1977], A note on approximation of the identity, Studia Math. 55, 111-122.

[1975], Pointwise convergence for integrable functions, Ph. D. Dissertation, Univ. of Minnesota, Minneapolis, Minn.

A. Zygmund [1959], Trigonometric series, vols. 1 and 2, Cambridge Univ. Press, London. 189-201.

[1974], On Fourier coefficients and transforms of two variables, Studia Math. 50,

Department of Mathematics, Princeton University, Princeton, New Jersey 08540

Department of Mathematics, University of Wisconsin, Madison, Wisconsin 53706 